\title{
MULTIPLICATIVE RIEMANN INTEGRATION IN NORMED RINGS(1)
}

\author{
BY
}

P. R. MASANI

Table of Contents

CHAPTER $\quad$ PAGE

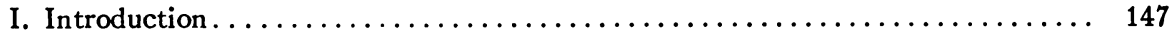

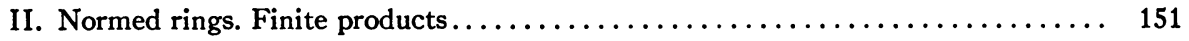

III. Definition of Riemann integration............................ 154

IV. Boundedness and the domain of integrability ...................... 161

V. Equivalence of additive and multiplicative integrability .............. 166

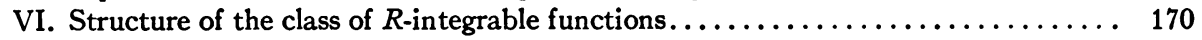

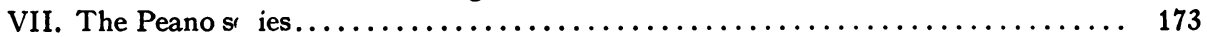

VIII. Differentiation......................................... 179

APPENDIX

I. A lemma concerning real integration. ........................... 188

II. An extension of Tannery's theorem........................... 189

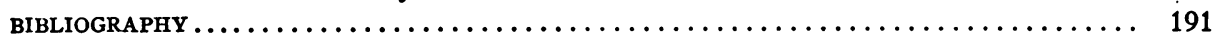

\section{INTRODUCTION}

1. Purpose of this paper. The purpose of this paper is to develop the theory of Riemann product integration of functions on real intervals with values in a normed ring (Banach algebra) $\left({ }^{2}\right)$.

Product integration of matrix-valued functions was initiated by V. Volterra $[1,2]\left(^{3}\right)$ in 1887 in connection with the theory of homogeneous linear differential equations. L. Schlesinger [1, 2] (1931) and G. Rasch [1] (1934) simplified and extended this theory by treating the matrices as linear algebras with normed topologies. In 1937 Garrett Birkhoff [2] remarked that the theory of product integration as developed by Schlesinger was applicable to normed rings $\left({ }^{4}\right)$. He also extended the theory of product integration to the nonlinear case.

There is no adequate treatment of the Riemann theory for discontinuous functions. Much remains to be done by way of strengthening the Riemann theory. In this paper we develop the Riemann theory for all integrable functions on a real interval with values in a normed ring. This extension is difficult in the infinite-dimensional case, for it is known (cf. L. Graves [1, p. 166])

Presented to the Society, August 23, 1946; received by the editors April 11, 1946.

(1) The writer wishes to make acknowledgment of the constant and valuable assistance received from Professor Garrett Birkhoff for this paper.

(2) Cf. Definition 3.1.

(8) Numbers in brackets refer to the bibliography at the end of the paper.

(4) Normed rings are complete normed linear algebras for which no restriction is made with regard to dimension. The matrices form normed rings of finite dimension. Cf. Definition 3.1. 
that in an infinite-dimensional normed ring everywhere discontinuous functions may be $R$-integrable and it is shown below $(\$ 20)\left({ }^{5}\right)$ that in such a ring the product of two $R$-integrable functions need not be $R$-integrable. In abandoning the assumption of continuity we are thus confronted with diffculties which never arise in ordinary integration.

We may now indicate the main new results we have established. After pointing out the duality between left and right product integration we show the equivalence of some previously employed notions of Riemann integration $(\$ \S 7,8)$. We then prove that every Riemann integrable function is bounded and that integrability on an interval implies integrability on every subinterval $(\$ \S 11,12)$. For product integration these results are far from obvious. We then establish the equivalence of additive and multiplicative integrability (\$16). This provides a powerful tool for the study of product integration( $\left.{ }^{6}\right)$. Next we show that the class of all $R$-integrable functions on $[a, b]$ into a normed ring $X$ is a closed additive subgroup (which is also a closed subring only when $X$ is finite-dimensional) of the ring of all bounded functions on $[a, b]$ into $X$ under the uniform topology given by $|f-\phi|$ $=\sup _{a \leqq t \leqq b}|f(t)-\phi(t)|(\S \S 19,20)$. We then show the validity of the Peano series representation for all $R$-integrable functions (\$22). We also show that with regard to the "fundamental problem of the integral calculus" our theory is no weaker than the classical Riemann theory ( $\$ 28)$. We conclude by establishing rules for product integration by parts and product integration after substitution $(\$ \S 29,30)$.

2. Continuous matrix-valued functions. For comparison we shall briefly review the product integration of continuous matrix-valued functions. The theory is considerably simplified by the fact that continuous functions are (by the Heine-Borel theorem) bounded and uniformly continuous.

Consider a continuous $n \times n$ matrix-valued function $A$ on $[a, b]$. Its Riemann product integral is defined by

$$
\int_{a}^{b \sim}(I+A d t)=\lim _{x \downarrow} \prod_{k=1}^{n}\left\{I+A\left(t_{k}\right)\left|\Delta_{k}\right|\right\}=\lim _{x \downarrow} J(A, \pi)
$$

where $I$ is the identity matrix, $\pi$ is a partition of $[a, b]$ into subintervals $\Delta_{k}$, and $t_{k} \in \Delta_{k}$. The limit is obtained with regard to the norm $\left({ }^{7}\right)$ of the space of matrices by taking successive refinements of $\pi$.

(5) Cf. p. 172 of this paper. The counter-example is due to Garrett Birkhoff.

( ${ }^{\circ}$ ) Additive integration theory for normed rings involves questions outside the corresponding theory for Banach spaces, namely, those arising from ring multiplication.

(7) For an $n \times n$ matrix $A=\left\{a_{i j}\right\}$, Schlesinger [1, pp. 33-34] employed the norm $|A|$ $=n \cdot \max _{i j}\left|a_{i j}\right|$. He showed that if $A$ and $B$ are $n \times n$ matrices and $c$ a real or complex number then $|A+B| \leqq|A|+|B|,|A B| \leqq|A| \cdot|B|,|c A|=|c| \cdot|A|$. He also showed that under this norm the $n \times n$ matrix-space is complete. Another norm for the matrix-space has been proposed by J. Wedderburn [1]. 
We shall assume that a norm is selected under which the space of $n \times n$ matrices is complete. Then to show the product integrability of $A$ we need only show that $\left|J(A, \pi)-J\left(A, \pi^{\prime}\right)\right|<\epsilon$ for all refinements $\pi^{\prime}$ of a suitably chosen partition $\pi$. Accordingly suppose $\pi^{\prime}$ subdivides each subinterval $\Delta_{k}$ of $\pi$ into subintervals $\Delta_{k}^{\alpha}$. Then

$$
\left|J(A, \pi)-J\left(A, \pi^{\prime}\right)\right|=\left|\prod_{k=1}^{n}\left\{I+A\left(t_{k}\right)\left|\Delta_{k}\right|\right\}-\prod_{k=1}^{n} \prod_{\alpha}\left\{I+A\left(t_{k}^{\alpha}\right)\left|\Delta_{k}^{\alpha}\right|\right\}\right| .
$$

But since matrix multiplication is continuous it can be shown (cf. (4.43)) that

$$
\left|J(A, \pi)-J\left(A, \pi^{\prime}\right)\right| \leqq \exp (M(b-a)) \sum_{k}\left|\left\{I+A\left(t_{k}\right)\left|\Delta_{k}\right|\right\}-\prod_{\alpha}\left\{I+A\left(t_{k}^{\alpha}\right)\left|\Delta_{k}^{\alpha}\right|\right\}\right|,
$$

where $M=\sup _{a \leqq t \leqq b}|f(t)|$. But

$$
\begin{aligned}
\mid\left\{I+A\left(t_{k}\right)\left|\Delta_{k}\right|\right\}-\prod_{\alpha}\{I & \left.+A\left(t_{k}^{\alpha}\right)\left|\Delta_{k}^{\alpha}\right| .\right\}|\leqq| A\left(t_{k}\right)\left|\Delta_{k}\right|-\sum_{\alpha} A\left(t_{k}^{\alpha}\right)\left|\Delta_{k}^{\alpha}\right| \mid \\
& +\left|I+\sum_{\alpha} A\left(t_{k}^{\alpha}\right)\right| \Delta_{k}^{\alpha}\left|-\prod_{\alpha}\left\{I+A\left(t_{k}^{\alpha}\right)\left|\Delta_{k}^{\alpha}\right|\right\}\right| .
\end{aligned}
$$

Now $(I+A)(I+B)$ is close to $I+A+B$ when $A$ and $B$ are small. Making an induction on this result it can be shown (cf. (4.32)) that the second term on the R.H.S. (right-hand side) of this equation can be made less than $\epsilon\left|\Delta_{k}\right|$, where $\epsilon \rightarrow 0$ with $|\pi|\left(^{8}\right)$. Also the first term on the R.H.S. is less than or equal to $\left|\Delta_{k}\right|$ times the oscillation of $A$ in $\Delta_{k}$. Hence

$$
\left|J(A, \pi)-J\left(A, \pi^{\prime}\right)\right| \leqq \exp (M(b-a)) \cdot \sum_{k}\left\{\epsilon+\operatorname{Osc}\left(f, \Delta_{k}\right)\right\}\left|\Delta_{k}\right| .
$$

Since the function $A$ is uniformly continuous on $[a, b]$ we see that the R.H.S. approaches zero as successive refinements of $\pi$ are taken. Thus $A$ possesses a product integral.

Associated with this notion of product integral is the corresponding notion of product derivative $\left({ }^{9}\right)$,

$$
A^{\prime}(x)=\lim _{\Delta_{x} \rightarrow 0} \frac{1}{\Delta_{x}}\left\{A^{-1}(x) A\left(x+\Delta_{x}\right)-I\right\} .
$$

It is clear that if $Y$ is a product primitive (anti-derivative) of $A$ then

$$
Y^{\cdot}(x)=Y(x) A(x)
$$

where $Y^{\cdot}$ is the ordinary additive derivative.

(8) $|\pi|=\sup _{\Delta_{k} \in \pi}\left|\Delta_{k}\right|$.

(9) " $A^{-1}(x)^{n}$ abbreviates " $\{A(x)\}^{-1 "}$ when $\{A(x)\}^{-1}$ exists. 
Equation (3) brings out the connection between product integration of matrix-valued functions and the theory of ordinary linear differential equations. For a continuous matrix-valued function $A(t)=\left\{a_{i j}(t)\right\}$ let us consider the system of homogeneous linear differential equations

$$
\begin{gathered}
d y_{1} / d x=y_{1} a_{11}(x)+\cdots+y_{n} a_{n 1}(x), \\
\cdots+\cdots+y_{n} a_{n n}(x) .
\end{gathered}
$$

If $\left\{y_{11}(x), \cdots, y_{1 n}(x)\right\}, \cdots,\left\{y_{n 1}(x), \cdots, y_{n n}(x)\right\}$ be $n$ solutions such that $\operatorname{det}\left\{y_{i j}(x)\right\} \neq 0$ we call $\left\{y_{i j}(x)\right\}=Y(x)$ a matrix solution (cf. G. D. Birkhoff and R. E. Langer [1, pp. 42-44]) of the system (4). A matrix solution obviously satisfies

$$
Y \cdot(x)=Y(x) A(x) .
$$

Comparison with (3) at once reveals that the matrix solution is nothing but a product primitive (anti-derivative) of $A(x)$.

It is also easy to establish the reciprocity of product integration (1) and product differentiation (2). The reciprocity consists of the two statements:

(a) If $A$ is continuous and $Y(x)=\int_{a}^{x}(I+A d t)$ then $Y^{\prime}(x)=A(x)$, $a \leqq x \leqq b$.

(b) If the product derivative $B^{\prime}$ is continuous then

$$
B(x)=B(a) \int_{a}^{x \frown}\left(I+B^{\prime} d t\right), \quad a \leqq x \leqq b .
$$

From the last equation and the result (cf. L. Schlesinger [1, pp. 42-44])

$$
\operatorname{det} \int_{a}^{x \frown}(I+A d t)=\exp \left(\int_{a}^{x} \sum_{i} a_{i i}(t) d t\right)
$$

we see at once that the product integral of $A$ is a matrix solution of the system of differential equations (4). The most general matrix solution is obtained by multiplying the product integral of $A$ by an arbitrary nonsingular constant matrix $C$.

We shall now obtain an integral equation for the product integral $Y$ of a continuous matrix-valued function $A$. From (3) we have

$$
Y(x)-Y(a)=\int_{a}^{x} Y^{\cdot}(t) d t=\int_{a}^{x} Y(t) A(t) d t .
$$

Now since $Y$ is continuous, clearly $Y(a)=I$. We thus obtain the following integral equation for $Y$ :

$$
Y(x)=I+\int_{a}^{x} Y(t) A(t) d t
$$


It may be shown that the method of successive approximations applied to this equation gives the following infinite series representation $\left({ }^{10}\right)$ for the product integral of a continuous matrix-valued function $A$

$$
\begin{aligned}
\int_{a}^{x}(I+A d t)=I & +\int_{a}^{x} A\left(t_{1}\right) d t_{1}+\int_{a}^{x} \int_{a}^{t_{2}} A\left(t_{1}\right) A\left(t_{2}\right) d t_{1} d t_{2} \\
& +\int_{a}^{x} \int_{a}^{t_{3}} \int_{a}^{t_{2}} A\left(t_{1}\right) A\left(t_{2}\right) A\left(t_{3}\right) d t_{1} d t_{2} d t_{3}+\cdots
\end{aligned}
$$

Both the series representation (6) and the integral equation (5) are obtained under weaker assumptions in Chapter VII.

\section{Normed RINGs. Finite PRODUCTS}

3. The normed ring. We now turn to the exact discussion of the definition and properties of normed rings $\left({ }^{11}\right)$.

DEFINITION 3.1. $X$ is a normed ring if and only if:

(i) $\boldsymbol{X}$ is a complete normed linear (Banach $\left({ }^{12}\right)$ ) space with real or complex scalars;

(ii) $X$ is a linear associative algebra with unit 1 ;

(iii) (13) For all $x, y \in X,|x y| \leqq|x| \cdot|y|,|1|=1$.

The space $X$ is a complete metric space ${ }^{(14)}$ under the metric $(x, y)=|x-y|$. Hence the notions of Cauchy sequence, sequence tending to a limit, convergent infinite series, continuous function, and so on, are obvious for normed rings. So are many results on limits, continuity and infinite series. We shall merely state here a few results which will be employed in the subsequent discussion. Detailed study of analysis in normed rings will be found in the papers of J. von Neumann [1], K. Yosida [1], and I. Gelfand [1].

$y$ is called the inverse of $x$ if and only if $x y=1=y x$. From the associative law it at once follows that an element can have at most one inverse. The inverse of $\mathbf{x}$ will be denoted by $\mathbf{x}^{-1} . X$ may of course possess nonzero elements without inverses, but we have the following results.

(3.2) If $|x-1|<1$ then $x^{-1}=\sum_{n=0}^{\infty}(1-x)^{n}$ exists.

(3.3) If $|x-1|<1$ then $\left|x^{-1}\right| \leqq 1 /(1-|1-x|)$.

(3.4) $x^{-1}$ is a continuous function of $x$.

(10) This series is due to G. Peano [1].

(11) The normed ring was first defined by K. Yosida [1, pp. 8-10] on a suggestion from M. Nagumo.

(12) S. Banach $[1$, p. 53] calls it a $(B)$ space. It is vector space with real or complex scalars on which there is defined a real function $|x|$ such that $|0|=0,|x|>0$ if $x \neq 0,|x+y| \leqq|x|$ $+|y|,|c x|=|c| \cdot|x|, c$ being a scalar. Also the space is complete, that is, for any sequence $\left(x_{n}\right)_{n=1}^{\infty}, \lim _{m, n \rightarrow \infty}\left|x_{m}-x_{n}\right|=0$ implies that there exists $x$ such that $\lim _{n \rightarrow \infty}\left|x_{n}-x\right|=0$.

(13) All that is actually needed is that multiplication be continuous with respect to the norm and $|1|>0$. I. Gelfand [1, pp. 1-2] has shown that when this is the case it is possible to find an equivalent norm satisfying (iii).

(ii) In the sense of M. Frechét $[1$, p. 1]. 
The exponential and logarithmic functions are defined by the equations

$$
\begin{aligned}
\operatorname{Exp}(\mathrm{x}) & =\sum_{n=0}^{\infty} \mathrm{x}^{n} / n !, & \mathrm{x} \in \mathrm{X}, \\
\operatorname{Ln}(\mathrm{x}) & =\sum_{n=1}^{\infty}(-1)^{n-1}(\mathrm{x}-1)^{n} / n, & |\mathrm{x}-1|<1 .
\end{aligned}
$$

They have the following properties.

(3.5) They are continuous in their domains of definition.

$$
\begin{aligned}
& |\operatorname{Exp}(\mathbf{x})| \leqq \exp |\mathbf{x}| \text {, } \\
& \left\{\begin{array}{lr}
\operatorname{Exp}(\operatorname{Ln}(\mathrm{x}))=\mathrm{x}, & \text { if }|\mathrm{x}-1|<1, \\
\operatorname{Ln}(\operatorname{Exp}(\mathrm{x}))=\mathrm{x}, & \text { if }|\mathrm{x}-1|<\operatorname{Ln} 2 .
\end{array}\right.
\end{aligned}
$$

(3.8) If $x y=y x$ then

$$
\begin{aligned}
\operatorname{Exp}(x) \cdot \operatorname{Exp}(y) & =\operatorname{Exp}(x+y), \\
\operatorname{Ln}(x \cdot y) & =\operatorname{Ln}(x)+\operatorname{Ln}(y) .
\end{aligned}
$$

4. Estimates for finite products in $X$. In this section we shall state a number of estimates for finite products in a normed ring $X$. These estimates will serve as lemmas in the following discussion of integration. Many of them are simplifications for the case of a normed ring of results obtained for the nonlinear case by Garrett Birkhoff [1, pp. 112-116]. Others have been stated by L. Schlesinger [1]. We shall mostly leave the proofs to the reader. Throughout this section $\mathbf{x}, \boldsymbol{y}, \boldsymbol{z}, \boldsymbol{u}, \mathbf{x}_{k}, \boldsymbol{y}_{k}$, and so on, will denote the elements of a normed ring $X$ and 1 its multiplicative unit.

The following estimate provides a lower bound for the change in a product resulting from a change in one of its factors when all other factors are suffciently near 1 . This estimate plays a fundamental role in the theory of product integration.

(4.11) If each $\left|x_{k}\right| \leqq 1 / 2$, then

$$
\left|\prod_{k=1}^{n}\left(1+x_{k}\right) \cdot u-\prod_{k=1}^{n}\left(1+x_{k}\right) \cdot v\right| \geqq \exp \left(-2 \sum_{k=1}^{n}\left|x_{k}\right|\right) \cdot|u-v| \text {. }
$$

Proof. Since $|x y| \leqq|x| \cdot|y|$ we have $|x-x y| \geqq(1-|y|)|x|$. Hence

$$
\left|\left(1+x_{n}\right) u-\left(1+x_{n}\right) v\right| \geqq\left(1-\left|x_{n}\right|\right)|u-v| \text {. }
$$

Now since $0 \leqq\left|x_{n}\right| \leqq 1 / 2$ we have(15) $1-\left|x_{n}\right| \geqq \exp \left(-2\left|x_{n}\right|\right)$. Hence

$$
\left|\left(1+x_{n}\right) u-\left(1+x_{n}\right) v\right| \geqq \exp \left(-2\left|x_{n}\right|\right) \cdot|u-v| \text {. }
$$

(15) In order to prove that $1-x \geqq e^{-2 x}$ for $0 \leqq x \leqq 1 / 2$ we need only show that the function $f(x)=e^{2 x}-x e^{2 x}-1 \geqq 0$ on $[0,1 / 2]$. Now $f^{\prime}(x)=e^{2 x}(1-2 x) \geqq 0$ on $[0,1 / 2]$. Hence on $[0,1 / 2]$, $f(x) \geqq f(0)=0$. 
By repeated application of this result we arrive at the lemma. (Q.E.D.)

It is easy to see that the last result admits of the following generalization.

(4.12) If each $\left|x_{k}\right|,\left|y_{k}\right| \leqq 1 / 2$ then

$$
\begin{array}{r}
\left|\prod_{k=1}^{m}\left(1+x_{k}\right) \cdot u \cdot \prod_{k=1}^{n}\left(1+y_{k}\right)-\prod_{k=1}^{m}\left(1+x_{k}\right) \cdot v \cdot \prod_{k=1}^{n}\left(1+y_{k}\right)\right| \\
\geqq \exp \left(-2\left\{\sum_{k=1}^{m}\left|x_{k}\right|+\sum_{k=1}^{n}\left|y_{k}\right|\right\}\right) \cdot|u-v| .
\end{array}
$$

The next two results provide simple upper bounds for finite products.

$$
\begin{gathered}
\left|\prod_{k=1}^{n}\left(1+\mathrm{x}_{k}\right)-1\right| \leqq \exp \sum_{k=1}^{n}\left|\mathrm{x}_{k}\right|-1 . \\
\left|\prod_{k=1}^{n}\left(1+\mathrm{x}_{k}\right)\right| \leqq \exp \sum_{k=1}^{n}\left|\mathrm{x}_{k}\right|
\end{gathered}
$$

The next two results answer the question as to how closely the product $\prod_{1}^{n}\left(1+x_{k}\right)$ approximates to the sum $1+\sum_{1}^{n} x_{k}$ when the $x_{k}$ are small. This question is important in the study of the connection between additive and multiplicative integration.

$$
\begin{aligned}
\prod_{k=1}^{n}\left(1+x_{n}\right)-\left(1+\sum_{k=1}^{n} x_{k}\right) & =\sum_{k=1}^{n} x_{k}\left\{\prod_{i=k+1}^{n}\left(1+x_{i}\right)-1\right\} . \\
\left|\prod_{k=1}^{n}\left(1+x_{k}\right)-\left(1+\sum_{k=1}^{n} x_{k}\right)\right| & \leqq\left(\exp \sum_{k=1}^{n}\left|x_{k}\right|-1\right) \sum_{k=1}^{n}\left|x_{k}\right| .
\end{aligned}
$$

The next four results generalize the continuity of the product' $x y$ as a function of $x$ and $y$ to that of the product $x_{1} x_{2} \cdots x_{n}$ as a function of $\mathbf{x}_{1}, \mathbf{x}_{2}, \cdots, \mathbf{x}_{n}$.

$$
\prod_{k=1}^{n} x_{k}-\prod_{k=1}^{n} y_{k}=\sum_{k=1}^{n}\left\{\left(\prod_{i=1}^{k-1} y_{i}\right)\left(x_{k}-y_{k}\right)\left(\prod_{i=k+1}^{n} x_{i}\right)\right\}
$$

where $y_{0}=1=x_{n+1}$.

$$
\left|\prod_{k=1}^{n} x_{k}-\prod_{k=1}^{n} y_{k}\right| \leqq \sum_{k=1}^{n}\left|\prod_{i=1}^{k-1} y_{i}\right| \cdot\left|\prod_{i=k+1}^{n} x_{i}\right| \cdot\left|x_{k}-y_{k}\right|
$$

where $y_{0}=1=x_{n+1}$.

$$
\begin{aligned}
\mid \prod_{k=1}^{n}\left(1+x_{k}\right)-\prod_{k=1}^{n} & \left(1+y_{k}\right) \mid \\
& \leqq \exp \left(\sum_{k=1}^{n}\left(\left|x_{k}\right|+\left|y_{k}\right|\right)\right) \cdot \sum_{k=1}^{n}\left|x_{k}-y_{k}\right| .
\end{aligned}
$$




$$
\begin{aligned}
\left|\prod_{k=1}^{n}\left(1+x_{k}\right)-\prod_{k=1}^{n}\left(1+y_{k}\right)\right| \\
\leqq\left\{\begin{array}{l}
\exp \left(\sum_{k=1}^{n}\left|x_{k}\right|\right)\left[\exp \left(\sum_{k=1}^{n}\left|x_{k}-y_{k}\right|\right)-1\right], \\
\exp \left(\sum_{k=1}^{n}\left|y_{k}\right|\right)\left[\exp \left(\sum_{k=1}^{n}\left|x_{k}-y_{k}\right|\right)-1\right] .
\end{array}\right.
\end{aligned}
$$

This at once yields the following interesting corollary.

$$
\begin{aligned}
& \left|\prod_{k=1}^{n}\left(1+x_{k}+y_{k}\right)-\prod_{k=1}^{n}\left\{\left(1+x_{k}\right)\left(1+y_{k}\right)\right\}\right| \\
& \quad \leqq \exp \left(\sum_{k=1}^{n}\left(\left|x_{k}\right|+\left|y_{k}\right|\right)\right)\left\{\exp \left(\sum_{k=1}^{n}\left|x_{k}\right| \cdot\left|y_{k}\right|\right)-1\right\} .
\end{aligned}
$$

The next result proves useful in the study of the reciprocity of integration and differentiation.

If $Y_{0}=1$ and each $Y_{k}$ has an inverse then

$$
\begin{aligned}
& \left|\prod_{k=1}^{n}\left(1+\mathbf{x}_{k}\right)-Y_{n}\right| \\
& \quad \leqq \exp \left(\sum_{i=1}^{n}\left|\mathbf{x}_{i}\right|\right) \sum_{k=1}^{n}\left\{\left|Y_{k-1} \mathbf{x}_{k}-\left[Y_{k}-Y_{k-1}\right]\right| \exp \left(-\sum_{i=1}^{k}\left|\mathbf{x}_{i}\right|\right)\right\} .
\end{aligned}
$$

In conclusion the following result shows how closely $\prod_{1}^{n}\left(1+x_{k}\right) \cdot \prod_{n}^{1}\left(1-x_{k}\right)$ approximates to 1 when the $\mathbf{x}_{k}$ are small.

$$
\left|\prod_{k=1}^{n}\left(1+\mathrm{x}_{k}\right) \cdot \prod_{k=n}^{1}\left(1-\mathrm{x}_{n}\right)-1\right| \leqq \exp \left(2 \sum_{k=1}^{n}\left|\mathrm{x}_{k}\right|\right) \sum_{k=1}^{n}\left|\mathrm{x}_{k}\right|^{2} .
$$

\section{Definition of RiEmanN INTEgRation}

In this chapter we shall define the convergence of functions whose arguments are Riemann partitions of an interval and whose values lie in a normed ring. We shall then define additive and multiplicative Riemann integration. After indicating the duality between left and right product integration, we shall show the equivalence of our definitions with some of the others given for Riemann integration. Finally with the aid of the notions of relative integral range and span we shall express the integrability conditions in a convenient form.

5. Partitions, functions, convergence. By a Riemann partition $\pi$ of a real interval $[a, b]$ we shall mean as usual a decomposition of $[a, b]$ into a finite set of nonoverlapping closed subintervals $\Delta_{1}, \cdots, \Delta_{n}$. Thus $\pi=\left\{\Delta_{1}, \cdots, \Delta_{n}\right\}$. By the norm $|\pi|$ of a partition $\pi$ we shall mean 
$\sup _{\Delta_{k} \in \pi}\left|\Delta_{k}\right| . n(\pi)$ will denote the number of subintervals in $\pi$. We shall say that $\pi^{\prime}$ is a subpartition of $\pi$ (briefly $\pi^{\prime}<\pi$ ) if $\Delta_{k}^{\prime} \in \pi^{\prime}$ implies the existence of $\Delta_{l} \in \pi$ such that $\Delta_{k}^{\prime} \subset \Delta_{l}$.

If $\pi=\left\{\Delta_{1}, \cdots, \Delta_{n}\right\}$ is a Riemann partition of $[a, b]$ then each set $\pi^{*}=\left\{t_{1}, \cdots, t_{n}\right\}$ where $t_{k} \in \Delta_{k}$ will be called a valuation of $\pi$. Each partition has of course an infinite number of valuations. Let us denote by $\Pi_{a}^{b}$ the class of all Riemann partitions of $[a, b]$. The theory of integration is largely concerned with many-valued functions from $\Pi_{a}^{b}$.into a normed ring $X$. Given, for example, a function' $\boldsymbol{f}$ on $[a, b]$ with values in $\boldsymbol{X}$, the approximative sum $J(\pi)=\sum_{k} f\left(t_{k}\right)\left|\Delta_{k}\right|$ is a function from $\Pi_{a}^{b}$ into $X$. It is a many-valued function since we can select each $t_{k}$ in different ways. It is however a single-valued function of $\pi$ and its valuation $\pi^{*}$. Thus

$$
J\left(\pi, \pi^{*}\right)=\sum_{\Delta_{k} \in \pi_{, t_{k}} \in \pi^{*}} f\left(t_{k}\right)\left|\Delta_{k}\right|
$$

and it seems to us that the exclusion of many-valued functions clarifies the theory.

We may now recall the definitions of Cauchy convergence and different limiting operations for functions $J\left(\pi, \pi^{*}\right)$ from $\Pi_{a}^{b}$ into $X$ and state some results showing their interrelationship.

Definition 5.1. We shall say that $J\left(\pi, \pi^{*}\right)$ is Cauchy convergent as $\pi \downarrow$ if and only if for all $\epsilon>0$ there exists $\pi_{\epsilon}$ such that $\pi<\pi_{\epsilon}$ implies, for all valuations $\pi^{*}, \pi_{e}^{*},\left|J\left(\pi, \pi^{*}\right)-J\left(\pi_{e}, \pi_{\epsilon}^{*}\right)\right|<\epsilon$.

Definition 5.2. We shall say that $\lim _{x \downarrow} J\left(\pi, \pi^{*}\right)=L$ if and only if for all $\epsilon>0$ there exists $\pi_{\epsilon}$ such that $\pi<\pi_{\epsilon}$ implies, for all valuations $\pi^{*}$, $\left|J\left(\pi, \pi^{*}\right)-L\right|<\epsilon$.

Definition 5.3. We shall say that $\lim _{|\pi| \rightarrow 0} J\left(\pi, \pi^{*}\right)=L$ if and only if for all $\epsilon>0$ there exists $\delta_{\epsilon}>0$ such that $|\pi|<\delta_{\epsilon}$ implies for all valuations $\pi^{*}$, $\left|J\left(\pi, \pi^{*}\right)-L\right|<\epsilon$.

DEFINITION 5.4. If $\left(\pi_{n}\right)_{n=1}^{\infty}$ be a sequence such that $\lim _{n \rightarrow \infty}\left|\pi_{n}\right|=0$ we shall say that $\lim _{n \rightarrow \infty} J\left(\pi_{n}, \pi_{n}^{*}\right)=L$ if and only if for all $\epsilon>0$ there exists a positive integer $n_{\mathrm{e}}$ such that $n \geqq n_{\varepsilon}$ implies for all $\pi_{n}$ and all valuations $\pi_{n}^{*}$, $\left|J\left(\pi_{n}, \pi_{n}^{*}\right)-L\right|<\epsilon$.

We note that the class $\Pi_{a}^{b}$ is directed by the relation $\prec$. For if $\pi_{1} \cdot \pi_{2}$ is the product partition obtained by the superposition of $\pi_{1}$ and $\pi_{2}$, we always have $\pi_{1} \cdot \pi_{2}<\pi_{1}, \pi_{2}$. Also, as already observed, $X$ is a complete metric space under the topology of its norm. Hence as is well known in general analysis $\left({ }^{16}\right)$ :

(5.5) Every Cauchy convergent function has a limit.

Again since $X$ is a Hausdorff space we have (cf. Garrett Birkhoff [3, p. 31]):

(5.6) The function $J\left(\pi, \pi^{*}\right)$ converges to at most one limit.

(16) Cf. E. H. Moore and H. L. Smith [1, pp. 106-107]. They consider numerically valued functions, but their proof clearly applies to functions with values in a complete metric space. 
6. Definition of Riemann integration. For any given function $\boldsymbol{f}$ on $[a, b]$ into a normed ring $X$, we define the following functions of $\pi$ and $\pi^{*}$.

$$
\begin{aligned}
& J \frown\left(f, \pi, \pi^{*}\right)=\prod_{k=1, \Delta_{k} \in t_{,} \in t_{k} \in \pi^{*}}^{k=n}\left\{1+f\left(t_{k}\right)\left|\Delta_{k}\right|\right\}, \\
& \mathcal{J}\left(\boldsymbol{f}, \pi, \pi^{*}\right)=\prod_{k=n, \Delta_{k} \in \prod_{x, t_{k} \in \pi^{*}}^{k=1}}\left\{1+f\left(t_{k}\right)\left|\Delta_{k}\right|\right\}, \\
& J\left(f, \pi, \pi^{*}\right)=\sum_{k=1, \Delta_{k} \in x_{,} t_{k} \in \pi^{*}}^{k=n} f\left(t_{k}\right)\left|\Delta_{k}\right| .
\end{aligned}
$$

We shall call these the left approximative product, the right approximative product, and the approximative sum respectively. We shall of ten abbreviate the sum on the R.H.S. of the last equation to $\sum_{1}^{n} f\left(t_{k}\right)\left|\Delta_{k}\right|$ and similarly with the two products.

Using these functions we can easily define the Riemann integrability of $f$. We have included a definition of additive integrability since a comparative study of additive and multiplicative integration proves useful $\left({ }^{17}\right)$.

Definition 6.1. If $\boldsymbol{f}$ is a function on $[a, b]$ into $\boldsymbol{X}$, we shall say that $\boldsymbol{f}$ is $R$-integrable on $[a, b]$ if and only if $\lim _{\pi \downarrow} J^{-}\left(f, \pi, \pi^{*}\right)$ exists. The limit will be called the left multiplicative (or product) Riemann integral of $f$ from $a$ to $b$ and will be denoted by $\int_{a}^{b} \frown(1+f d t)$. We give a corresponding definition

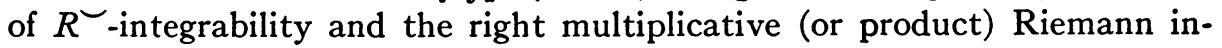

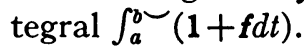

Definition 6.2. If $f$ is a function on $[a, b]$ into $X$ we shall say that $f$ is $R$-integrable on $[a, b]$ if and only $\lim _{\pi \downarrow} J\left(f, \pi, \pi^{*}\right)$ exists. The limit will be called the additive Riemann integral of $f$ from $a$ to $b$ and will be denoted by $\int_{a}^{b} f d t$.

7. Duality. Since multiplication in $X$ is in general noncommutative, it follows that in general $\int_{a}^{b}(1+f d t) \neq \int_{a}^{b} \frown(1+f d t)$. We shall now show, however, that a duality subsists between the two product integrals which renders unnecessary the statement and proof of corresponding theorems for both.

Suppose a class $A$ is a normed ring $\boldsymbol{X}$ under a certain norm and under certain algebraic operations. Let the ring multiplication operation be denoted by $(\cdot)$. Let us now define a new operation (o) as follows: $x \circ y=y \cdot x$. It is clear that $A$ becomes a different normed ring $X^{*}$ under the same norm and the same operations of addition and scalar multiplication, but with (0) instead of $(\cdot)$ as ring multiplication. The ring $X^{*}$ is called the dual of $X$, since obviously $\left(X^{*}\right)^{*}=X$. When $X$ is commutative we of course have $X^{*}=X$.

(17) The additive Riemann integral was first defined for functions with values in a Banach space by L. Graves $[1$, p. 166] in 1927. The multiplicative Riemann integral was first defined for matrix-valued functions by V. Volterra [2] in 1887 and with respect to a normed topology by L. Schlesinger [1, pp. 36-37] in 1931. 
Let us now make the following definitions.

Definition 7.1 (Dual limit). Let $L$ be the limit of a function $U(\alpha)$ with values in a normed ring $X$ with respect to the parameter $\alpha$, so that $L=\lim _{\alpha} U(\alpha)$.

(i) Suppose the expression abbreviated by " $U(\alpha)$ " involves finite products but no limits (with respect to other parameters $\beta, \gamma$, and so on). Let us rewrite this expression reversing every finite product. Let " $U^{*}(\alpha)$ " abbreviate the resulting expression. Then $\lim _{\alpha} U^{*}(\alpha)$ is called the dual of $L$ and is denoted by " $L$ "."

(ii) Suppose the expression abbreviated by " $U(\alpha)$ " involves finite products and/or limits (with respect to other parameters $\beta, \gamma$, and so on). Let us rewrite this expression reversing every finite product and replacing every limit by its dual (cf. (i)). Let " $U^{*}(\alpha)$ " abbreviate the resulting expression. Then $\lim _{\alpha} U^{*}(\alpha)$ is called the dual of $L$ and is denoted by " $L^{*}$."

This recursive definition completely defines the notion of "dual limit."

Definition 7.2 (Dual statement). Let $C$ be a statement involving finite products of elements of a normed ring $X$ and limits (with respect to certain parameters) of functions with values in $X$. Let us rewrite $C$ reversing every finite product and replacing every limit by its dual. The resulting statement is called the dual of $C$ and is denoted by $C^{*}$.

It is clear that $C$ is a true assertion regarding the ring $X$ if and only if $C^{*}$ is a true assertion regarding its dual $X^{*}$. Now suppose that $C$ has been generally established so that it holds for all normed rings ((4.11), for example, is such a $C$ ). Then $C$ must hold for both $X$ and $X^{*}$. Consequently $C^{*}$ must hold for $\boldsymbol{X}$. Thus both $C$ and $C^{*}$ are true assertions concerning $\boldsymbol{X}$. The following metatheorem expresses this fact in a convenient form.

Metatheorem (Duality Principle). Let $C$ be a statement involving products (either finite or infinite), product integrals and limits with respect to other parameters. If $C$ is generally established for all normed rings then both $C$ and its dual $C^{*}$ are (true) theorems.

In view of this principle we shall state and prove results only for left products and left product integrals.

8. Equivalence of limiting operations on $\pi$. According to definitions 6.1 and 6.2 , the multiplicative and additive integrals are limits as $\pi \downarrow$. In this section we shall sketch the argument to show that it would have made no difference had we construed them as limits as $|\pi| \rightarrow 0$ or as limits obtained sequentially (cf. Definitions 5.2-5.4).

The class $\Pi_{a}^{b}$ of all Riemann partitions of $[a, b]$ is directed by the relation $R: \pi R \pi^{\prime}$ if and only if $|\pi| \leqq\left|\pi^{\prime}\right|$. The equivalence of the limiting operations 5.3 and 5.4 therefore follows, as is well known in general analysis $\left({ }^{18}\right)$.

(18) Cf. E. H. Moore and H. L. Smith [1, p. 108, Proposition 8(B)]. They deal with real functions but their proof applies to functions with values in a metric space. 
Thus

(8.1) $\lim _{|\pi| \rightarrow 0} J\left(\pi, \pi^{*}\right)=L$ if and only if for every sequence $\left(\pi_{n}\right)_{n=1}^{\infty}$ such that $\lim _{n \rightarrow \infty}\left|\pi_{n}\right|=0$ we have $\lim _{n \rightarrow \infty} J\left(\pi_{n}, \pi_{n}^{*}\right)=L$.

Also since $\pi<\pi^{\prime}$ implies $|\pi| \leqq\left|\pi^{\prime}\right|$ we at once obtain

(8.2) $\lim _{|\pi| \rightarrow 0} J\left(\pi, \pi^{*}\right)=L$ implies $\lim _{\pi \downarrow} J\left(\pi, \pi^{*}\right)=L$.

(8.1) and (8.2) hold for any arbitrary function from $\Pi_{a}^{b}$ into $X$. The converse of (8.2) is not however generally valid( $\left.{ }^{19}\right)$, but holds for the approximative functions $J^{\frown}\left(f, \pi, \pi^{*}\right), J\left(f, \pi, \pi^{*}\right)$ employed in the definitions of integration, provided the function $f$ is bounded. We shall outline the proof, first recalling the definition of oscillation of a function.

Definition 8.3. If $\boldsymbol{f}$ is a function on $[a, b]$ into $\boldsymbol{X}$ and $[\xi, \zeta] \subset[a, b]$, then $\sup _{\xi \leq x, y \leq \zeta}|f(x)-f(y)|$ will be called the oscillation of $f$ in $[\xi, \zeta]$ and denoted by Osc $(f,[\xi, \zeta])$.

We shall leave the proof of the next result to the reader. It is a direct consequence of (4.32).

LEMMA 8.4. If $\boldsymbol{f}$ is a bounded function on $[a, b]$ and $M=\sup _{a \leq x \leq b}|\boldsymbol{f}(x)|$ and $\eta \in[\xi, \zeta] \subset[a, b]$ then

$$
\left|J \frown\left(f, \pi, \pi^{*}\right)-\{1+f(\eta)(\zeta-\xi)\}\right| \leqq(\zeta-\xi)[M(\exp (M(\zeta-\xi))-1)+\operatorname{Osc}(f,[\xi, \zeta]) .
$$

Let now $\pi, \pi_{0} \in \Pi_{a}^{b}, \pi^{*}$ be a valuation of $\pi$ and let $\left(\pi \cdot \pi_{0}\right)^{*}$ be that valuation of the product partition $\pi \cdot \pi_{0}$ which contains as many points of $\pi^{*}$ as possible. Using the last lemma it may be shown $\left({ }^{20}\right)$ that

$$
\left|J \frown\left(f, \pi, \pi^{*}\right)-J \frown\left(f, \pi \cdot \pi_{0},\left(\pi \cdot \pi_{0}\right)^{*}\right)\right| \leqq 2 \exp (2 M(b-a)) M \cdot n\left(\pi_{0}\right) \cdot|\pi| \cdot
$$

We may therefore draw the following conclusion. Given any bounded function $f$, any partition $\pi_{0}$ and any $\epsilon>0$ we can find $\delta_{\epsilon, \pi_{0}}>0$ such that there is a subpartition of $\pi_{0}$ for which the approximative product differs from that of any partition $\pi$ with $|\pi| \leqq \delta_{\epsilon, \pi_{0}}$ by less than $\epsilon$. Using the triangle inequality in conjunction with this result we obtain (8.5), which we had set out to prove.

(8.5) $\lim _{\pi \downarrow} J^{\frown}\left(f, \pi, \pi^{*}\right)=L$ implies $\lim _{|\pi| \rightarrow 0} J^{\frown}\left(f, \pi, \pi^{*}\right)=L$, provided the function $f$ is bounded( $\left.{ }^{21}\right)$.

Correspondingly for $R$-integration it is possible to establish the following result by essentially classical methods $\left({ }^{22}\right)$. We shall leave this to the reader.

(19) The following counter-example shows this. Consider Riemann partitions $\pi$ of $[0,1]$. Let " $x \in \pi$ " mean " $x$ is a point of subdivision of $\pi$," and let $i$ be any positive integer. Define

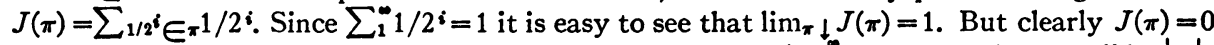
for a partition which excludes all members of the sequence $\left(1 / 2^{i}\right)_{i=1}^{\infty}$ no matter how small be $|\pi|$. Hence $\lim _{|\pi| \rightarrow 0} J(\pi) \neq 1$. Setting $J\left(\pi, \pi^{*}\right)=J(\pi)$ for all $\pi^{*}$, we have a counter-example.

(20) The proof is laborious.

(21) The restriction that $f$ is bounded is only temporary, for we shall see in the next chapter (Theorems 11.5 and 11.6) that every function for which $\lim _{x \downarrow} J^{-}\left(f, \pi, \pi^{*}\right)$ or $\lim _{\pi \downarrow} J\left(f, \pi, \pi^{*}\right)$ exists is bounded.

(22) In classical analysis, the result corresponding to (8.6) is known as Darboux's theorem. Cf. Goursat [1, p. 151]. 
(8.6) $\lim _{\pi \downarrow} J\left(f, \pi, \pi^{*}\right)=L$ implies $\lim _{|\pi| \rightarrow 0} J\left(f, \pi, \pi^{*}\right)=L$, provided the function $f$ is bounded( $\left.{ }^{21}\right)$.

We may summarise the results of this section in the following theorem.

THEOREM 8.7. For any bounded $\left.{ }^{21}\right)$ function $\boldsymbol{f}$, criteria of $R$-integrability according to definitions 5.2, 5.3 and 5.4 are equivalent. The same is true of $R$-integrability.

9. Relative integral range. It is well known that in the real case the condition: for some $\pi$, the upper and lower approximative sums $J^{*}(f, \pi), J_{*}(f, \pi)$ differ by less than $\epsilon$, or briefly $\inf _{\pi}\left\{J^{*}(f, \pi)-J_{*}(f, \pi)\right\}=0$, is sufficient for the $R$-integrability of $f\left({ }^{23}\right)$. We shall now formulate a similar condition for the general case of a function on $[a, b]$ with values in any normed ring $\boldsymbol{X}$. We must first introduce the notions of relative integral range and the diameter of a subset of $\boldsymbol{X}$.

Definition 9.1. If $\boldsymbol{f}$ is a function on $[a, b]$ into $X$, the set of all $J\left(f, \pi, \pi^{*}\right)$ obtainable for a given $\pi$ will be called the integral range of $f$ relative to $\pi$ and denoted by $S(f, \pi)$. Similarly we define $S^{\sim}(f, \pi)$ as the set of all $J^{\frown}\left(f, \pi, \pi^{*}\right)$ where $\pi^{*}$ is any valuation of $\pi$.

Definition 9.2. If $\boldsymbol{B} \subset \boldsymbol{X}$ then the positive real number $\sup _{\mathbf{x}, \boldsymbol{y} \in \boldsymbol{B}}|\mathbf{x}-\boldsymbol{y}|$ will be called the diameter of $B$ and denoted by $|\boldsymbol{B}|$.

In the real case we have for all valuations $\pi^{*}$ of $\pi, J_{*}(f, \pi) \leqq J\left(f, \pi, \pi^{*}\right)$ $\leqq J^{*}(f, \pi)$ and therefore $|S(f, \pi)|=J^{*}(f, \pi)-J_{*}(f, \pi)$. We thus arrive at the condition: $\inf _{\pi}|S(f, \pi)|=0$.

We shall now show that even under general conditions this condition is both necessary and sufficient for $R$-integrability. For this purpose we must define the convex hull of a subset of $X$.

Definition 9.3. If $B \subset X$, the set of all elements of the type $c_{1} x_{1}+\cdots$ $+c_{r} x_{r}$ where $x_{k} \in B$, each $c_{k} \geqq 0$ and $\sum_{1}^{r} c_{k}=1$ will be called the convex hull of $B$ and denoted by $B$ -

Next defining, for $B_{k} \subset X, \sum_{1}^{r} B_{k}$ as the set of elements $x_{1}+\cdots+x_{r}$ where $\mathbf{x}_{k} \in \boldsymbol{B}_{k}$ and $c \boldsymbol{B}_{k}$ or $\boldsymbol{B}_{k} c$ as the set of elements $c \mathbf{x}$ where $\mathbf{x} \in \boldsymbol{B}_{k}$, we can state the following important results $\left({ }^{24}\right)$.

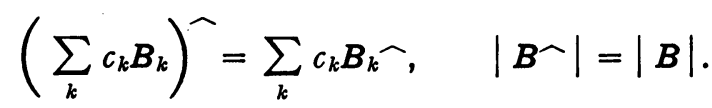

It should be observed that with this notation

$$
S(f, \pi)=\sum_{\pi} f\left(\Delta_{k}\right)\left|\Delta_{k}\right|
$$

where $f\left(\Delta_{k}\right)$ is the range of $f$ on $\Delta_{k}$.

Now let $\pi_{2}=\left\{\Delta_{1}, \cdots, \Delta_{n}\right\}$ and $\pi_{1}<\pi_{2}$, and suppose $\pi_{1}$ subdivides each

(23) Cf. for instance Goursat $[1$, p. 147].

(2) For proofs we would refer the reader to Garrett Birkhoff [1, pp. 359-360]. 
$\Delta_{k}$ into subintervals $\Delta_{k}^{\alpha}$. Then for all valuations $\pi_{1}^{*}$ we have

$$
\sum_{\alpha} f\left(t_{k}^{\alpha}\right) \frac{\left|\Delta_{k}^{\alpha}\right|}{\left|\Delta_{k}\right|} \in\left(f\left(\Delta_{k}\right)\right)
$$

since $f\left(t_{k}^{\alpha}\right) \in f\left(\Delta_{k}\right)$ and

$$
\sum_{\alpha} \frac{\left|\Delta_{k}^{\alpha}\right|}{\left|\Delta_{k}\right|}=1
$$

Hence

$$
J\left(f, \pi_{1}, \pi_{1}^{*}\right)=\sum_{k=1}^{n}\left\{\sum_{\alpha} f\left(t_{k}^{\alpha}\right) \frac{\left|\Delta_{k}^{\alpha}\right|}{\left|\Delta_{k}\right|}\right\}\left|\Delta_{k}\right| \in \sum_{k=1}^{n}\left(f\left(\Delta_{k}\right)\right)-\left|\Delta_{k}\right| .
$$

But from (9.4) and (9.5)

$$
\sum_{k=1}^{n}\left(f\left(\Delta_{k}\right)\right)-\left|\Delta_{k}\right|=\left(\sum_{k=1}^{n} f\left(\Delta_{k}\right)\left|\Delta_{k}\right|\right)^{\widehat{ }}=\left(S\left(f, \pi_{2}\right)\right) \uparrow .
$$

Thus

(9.6) $\pi_{1} \prec \pi_{2}$ implies $J\left(f, \pi_{1}, \pi_{1}^{*}\right) \in\left(S\left(f, \pi_{2}\right)\right)$ -

Suppose now that $\inf _{\pi}|S(f, \pi)|=0$ so that given any $\epsilon>0$ there exists $\pi_{\epsilon}$ such that $\left|S\left(f, \pi_{\epsilon}\right)\right|<\epsilon$. If $\pi<\pi_{\epsilon}$ and $\pi^{*}, \pi_{\epsilon}^{*}$ be valuations then we have

$$
\begin{aligned}
J\left(f, \pi, \pi^{*}\right) & \in\left(S\left(f, \pi_{\epsilon}\right)\right) \\
J\left(f, \pi_{\epsilon}, \pi_{\epsilon}^{*}\right) & \in S\left(f, \pi_{\epsilon}\right) \subset\left(S\left(f, \pi_{\epsilon}\right)\right)-.
\end{aligned}
$$

Hence from (9.4)

$$
\left|J\left(f, \pi, \pi^{*}\right)-J\left(f, \pi_{\epsilon}, \pi_{\epsilon}^{*}\right)\right| \leqq\left|\left(S\left(f, \pi_{\epsilon}\right)\right)-\right|=\left|S\left(f, \pi_{\epsilon}\right)\right|<\epsilon .
$$

It follows that $f$ is $R$-integrable. Conversely if $f$ is $R$-integrable then obviously inf $\boldsymbol{x}_{\boldsymbol{\pi}}|\mathbf{S}(\boldsymbol{f}, \pi)|=0$. Thus:

LemMA 9.7. $f$ is R-integrable if and only if $\inf _{\pi}|S(f, \pi)|=0$.

10. Relative integral span. In this section we shall attempt to do for the product integral what was done in the last section for the additive case.

It should first be observed that the condition $\inf _{\pi}\left|S^{\sim}(f, \pi)\right|=0$ is not sufficient for $R$-integrability. For consider the real function $f$ defined in $[0,1]$ as follows

$$
f(0)=0, \quad f(x)= \begin{cases}1 / x-2, & 0<x<1 / 2, \\ -2, & 1 / 2 \leqq x \leqq 1 .\end{cases}
$$

Let $\pi_{\epsilon}$ be any partition with $\Delta_{k}=[1 / 2,1]$ as subinterval. Then for all valua- 
tions $\pi_{\epsilon}^{*}, \pi_{\epsilon}^{\dagger}$ we have $J \frown\left(f, \pi_{\epsilon}, \pi_{\epsilon}^{*}\right)=0=J \frown\left(f, \pi_{\epsilon}, \pi_{\epsilon}^{\dagger}\right)$ for both products contain the factor $1+(-2)\left|\Delta_{k}\right|=0$. Hence $\left|S \backsim\left(f, \pi_{\epsilon}\right)\right|=0$. But since $f$ is unbounded it is not $R$-integrable on $[0,1]$ (cf. Theorem 11.5).

Since the reals form a (topological) subring of every normed ring $X$, it follows that there is no normed $X$ for which the condition $\inf _{\pi}\left|S^{\sim}(f, \pi)\right|=0$ is sufficient for $R$-integrability in $X$. We shall see later (cf. Theorem 16.4) that the slightly stronger condition $\lim _{x_{0} \downarrow} \inf _{x_{2}}<\pi_{0}|S \frown(f, \pi)|=0$ is sufficient for $R$-integrability. For the present we note that it is obviously necessary; thus:

Lemma 10.1. If $f$ is $R$-integrable then $\lim _{\pi_{0} \downarrow} \inf _{\pi}<\pi_{0}|S \frown(f, \pi)|=0$.

A necessary and sufficient condition for $R$-integrability similar to that given in Lemma 9.7 is however needed to simplify the proofs in the next chapter and to keep clear the parallelism between additive and multiplicative integration. We obtain such a condition by extending the notion of relative integral range to what we have termed relative integral span and then reformulating the definition 6.1 of $R$-integrability.

Definition 10.2. If $\boldsymbol{f}$ is a function on $[a, b]$ with values in $X$, the set of all $J^{-}\left(f, \pi^{\prime}, \pi^{*}\right)$ obtainable for all subpartitions $\pi^{\prime}$ of a given $\pi$ and all valuations $\pi^{* *}$ will be called the integral span of $f$ relative to $\pi$ and denoted by $T \frown(f, \pi)$.

Definition 10.3. The positive number

$$
\sup _{\boldsymbol{\pi}^{\prime}<\pi_{, \pi^{\prime}, \pi^{*}}}\left|J \frown\left(f, \pi^{\prime}, \pi^{\prime *}\right)-J \frown\left(f, \pi, \pi^{*}\right)\right|
$$

will be called the modulus of $T^{\sim}(f, \pi)$ and denoted by $\left\|T^{\sim}(f, \pi)\right\|$.

It should be observed that $\left\|T^{\sim}(f, \pi)\right\| \leqq\left|T^{\sim}(f, \pi)\right|$ and that from the triangle inequality it follows that $\pi_{1}<\pi_{2}$ implies $\left\|T^{\sim}\left(f, \pi_{1}\right)\right\| \leqq 2\left\|T^{\sim}\left(f, \pi_{2}\right)\right\|$. From definition 6.1 we at once have:

LEMMA 10.4. $f$ is $R$-integrable if and only if inf $\|\boldsymbol{T}(\boldsymbol{f}, \pi)\|=0$.

\section{BOUNDEDNESS AND THE DOMAIN OF INTEGRABILITY}

In this chapter we shall prove the boundedness of all (properly) Riemann integrable functions and show that if a function is integrable on an interval it is integrable on all its subintervals. We shall then show that every product integral possesses an inverse.

11. Boundedness of integrable functions. While the proof that every $R$-integrable function is bounded is trivial, the proof of the corresponding result for $R$-integration goes deeper (cf. the beginning of $\$ 10)$. We have to appeal to the Heine-Borel theorem and then to (4.12), which plays a vital role in the theory of $R$-integration. We must first establish the following combinatorial lemma.

(11.1) Let $\mathcal{N}=\left\{\sigma_{x_{1}}, \cdots, \sigma_{x_{m}}\right\}$ be a finite covering of $[a, b]$ where $\sigma_{x_{k}}$ 
is a closed subinterval not wholly contained in any other such subinterval and $x_{k} \in \sigma_{x_{k}}$. Then there exists $\pi=\left\{\Delta_{1}, \cdots, \Delta_{m}\right\} \in \Pi_{a}^{b}$ such that $x_{k} \in \Delta_{k} \subset \sigma_{x_{k}}$.

Proof. We shall use induction. The result is obvious for $m=1$. Next let us assume that from every covering of the given type consisting of $m$ subintervals we can construct a Riemann partition with the required properties. Consider then any covering $\mathcal{N}=\left\{\sigma_{x_{1}}, \cdots, \sigma_{x_{m}}, \sigma_{x_{m+1}}\right\}$ of $[a, b]$ of the given type where we assume that $a \leqq x_{1}<\cdots<x_{m}<x_{m+1} \leqq b$. Let $y_{m}$ be the largest right end point of $\sigma_{x_{k}}, k=1, \cdots, m$. There are now two possibilities, (i) $x_{m+1} \in\left[y_{m}, b\right]$ or (ii) $x_{m+1} \in\left[a, y_{m}\right]$.

In case $(\mathrm{i}),\left[y_{m}, b\right] \subset \sigma_{x_{m+1}}$. Also $\left\{\sigma_{x_{1}}, \cdots, \sigma_{x_{m}}\right\}$ is a covering of $\left[a, y_{m}\right]$ and by our assumption we can obtain a partition $\left\{\Delta_{1}, \cdots, \Delta_{m}\right\}$ of $\left[a, y_{m}\right]$ having the required properties. Add to this partition the subinterval $\Delta_{m+1}=\left[y_{m}, b\right] \subset \sigma_{x_{m+1}}$ and we obtain a partition of $[a, b]$ with the required properties.

In case (ii), $\left\{\sigma_{x_{1}}, \cdots, \sigma_{x_{m}}\right\}$ is a covering of $\left[a, x_{m+1}\right]$ of the given type since $x_{k}<x_{m+1}, k=1, \cdots, m$. Again by our assumption we have a partition $\pi$ of $\left[a, x_{m+1}\right]$ with the required properties. Also obviously $x_{m+1} \in\left[x_{m+1}, b\right]$ $C \sigma_{x_{m+1}}$. Hence adding to $\pi$ the subinterval $\Delta_{m+1}=\left[x_{m+1}, b\right]$ we obtain a partition of $[a, b]$ having the required properties.

The result thus follows by induction. (Q.E.D.)

(11.2) If $K(x)$ is a positive function on $[a, b]$ then there exists $\pi_{0}=\left\{\Delta_{1}, \cdots, \Delta_{n}\right\} \in \Pi_{a}^{b}$ such that each $\Delta_{k}$ contains a point $x_{k}$ with the property $\left|\Delta_{k}\right| \leqq K\left(x_{k}\right)$.

Proof. Surround each point $x \in[a, b]$ by a closed subinterval $\sigma_{x}$ of length $\left|\sigma_{x}\right| \leqq K(x)$ and such that $x$ is the middle point of $\sigma_{x}$ unless $x=a$ or $x=b$ (in which case $x$ is respectively the left or right end point of $\sigma_{x}$ ). The family $\mathcal{N}$ of these subintervals is a covering of $[a, b]$. By the Heine-Borel theorem we can extract a finite covering $\mathcal{N}_{0}=\left\{\sigma_{x_{1}}, \cdots, \sigma_{x_{n}}\right\} \subset \mathcal{N}$.

Clearly $x_{k} \in \sigma_{x_{k}}$ and $\left|\sigma_{x_{k}}\right| \leqq K\left(x_{k}\right)$ and we may assume that no interval $\sigma_{x_{k}}$ is wholly contained in another such interval. Applying the last result we can construct a partition $\pi=\left\{\Delta_{1}, \cdots, \Delta_{n}\right\} \in \Pi_{a}^{b}$ such that $x_{k} \in \Delta_{k} \subset \sigma_{x_{k}}$. Also $\left|\Delta_{k}\right| \leqq\left|\sigma_{x_{k}}\right| \leqq K\left(x_{k}\right)$. (Q.E.D.)

(11.3) For all $\pi_{0}$ there exists $\pi<\pi_{0}$ such that for every $\Delta_{i} \in \pi$

$$
|S \frown(f, \pi)| \geqq \exp (-n(\pi)) \cdot \operatorname{Osc}\left(f, \Delta_{i}\right)\left|\Delta_{i}\right| \text {. }
$$

Proof. Let $\pi_{0}=\left\{\sigma_{1}, \cdots, \sigma_{m}\right\}$. With the help of (11.2) we construct a partition $\pi_{0}^{k}$ of each subinterval $\sigma_{k} \in \pi_{0}$ with the property that each $\Delta_{k}^{\alpha} \in \pi_{0}^{k}$ has within it a point $x_{k}^{\alpha}$ such that ${ }^{(25)}\left|\Delta_{k}^{\alpha}\right| \leqq 1 / 2\left|f\left(x_{k}^{\alpha}\right)\right|$. Let now $\left.{ }^{26}\right)$

$$
\pi=\pi_{0}^{1} \cup \ldots \cup \pi_{0}^{m}=\left\{\Delta_{1}, \cdots, \Delta_{n}\right\},
$$

${ }^{(25)}$ We construct a partition possessing this property so as to be able to apply the fundamental result (4.12).

${ }^{(28)}$ Here and throughout the sequel $\cup$ will denote set theoretic union. 
where the subintervals $\Delta_{k}$ are in their natural order from $a$ to $b$. Clearly $\pi<\pi_{0}$. Also there exist points $x_{1}, \cdots, x_{n}$ such that

$$
x_{k} \in \Delta_{k}, \quad\left|\Delta_{k}\right| \leqq 1 / 2\left|f\left(x_{k}\right)\right| .
$$

Now let $\pi^{*}, \pi^{\dagger}$ be valuations of $\pi$ which contain the points $x_{k}$ (satisfying (1)) from all subintervals of $\pi$ except $\Delta_{i}$ from which they contain different points $t_{i}, t_{i}{ }^{\prime}$. Then from the fundamental result (4.12) we have

$$
\begin{aligned}
\mid J \frown\left(f, \pi, \pi^{*}\right)- & J \frown\left(f, \pi, \pi^{\dagger}\right) \mid \\
& \geqq \exp \left(-2 \sum_{k \neq i}\left|f\left(x_{k}\right)\right|\left|\Delta_{k}\right|\right) \cdot\left|f\left(t_{i}\right)\right| \Delta_{i}\left|-f\left(t_{i}^{\prime}\right)\right| \Delta_{i}|| \\
& \geqq \exp (-n(\pi)) \cdot\left|f\left(t_{i}\right)-f\left(t_{i}^{\prime}\right)\right|\left|\Delta_{i}\right|
\end{aligned}
$$

in view of (1). Hence

$$
|S \frown(f, \pi)| \geqq \exp (-n(\pi)) \cdot\left|f\left(t_{i}\right)-f\left(t_{i}^{\prime}\right)\right|\left|\Delta_{i}\right| .
$$

Since this holds for all $t_{i}, t_{i}^{\prime} \in \Delta_{i}$ we get

$$
|S \frown(f, \pi)| \geqq \exp (-n(\pi)) \cdot \operatorname{Osc}\left(f, \Delta_{i}\right)\left|\Delta_{i}\right| \text {. }
$$

(Q.E.D.)

Suppose now that $f$ is unbounded on $[a, b]$; then by the Heine-Borel theorem there exists $x \in[a, b]$ such that for every neighbourhood $\Delta_{x}$ containing $x$, Osc $\left(f, \Delta_{x}\right)=\infty$. It follows from (11.3) that:

(11.4) $\lim _{\pi_{0} \downarrow} \inf _{\pi<\pi_{0}}\left|S^{\sim}(f, \pi)\right|=0$ implies $f$ is bounded.

In particular (cf. Lemma 10.1) we have:

THEOREM 11.5. Every $R$-integrable function is bounded.

The proof of the corresponding result for $R$-integration is trivial. Thus: (11.6) Every $R$-integrable function is bounded.

CoRollary 11.7. If $M=\sup _{a} \leqq t \leqq b|f(t)|$ then

$$
\left|\int_{a}^{b} f d t\right| \leqq M(b-a), \quad\left|\int_{a}^{b}(1+f d t)\right| \leqq \exp (M(b-a))
$$

(cf. (4.22)).

12. The domain of integrability. The question of the domain of integrability is closely related to the effect of contraction of a partition on the relative integral range and span. We define this notion $\left({ }^{27}\right)$.

Definition 12.1. (a) If $\pi=\left\{\Delta_{1}, \cdots, \Delta_{n}\right\} \in \Pi_{a}^{b}$ and $[c, d] \subset[a, b]$ then the partition of $[c, d]$ consisting of the subintervals $\left({ }^{28}\right) \Delta_{k} \cap[c, d]$ of positive

(27) Contraction corresponds to the notion of relativisation in topology.

${ }^{(28)}$ Here and throughout the sequel $\bigcap$ will denote set theoretic intersection. 
length is called the contraction of $\pi$ to $[c, d]$ and is denoted by $\pi_{c}^{d}$. (b) If $\pi^{*}$ be a valuation of $\pi \in \Pi_{a}^{b}$ and $\pi_{c}^{d}$ the contraction of $\pi$ to $[c, d]$, then the valuation $\pi_{c}^{d *}=\pi^{*} \cap[c, d]$ of $\pi_{c}^{d}$ is called the contraction of $\pi^{*}$ to $[c, d]$. We add $c$ and/or $d$ to $\pi_{c}^{d *}$ in case $\pi^{*} \cap[c, d]$ does not contain any points from the extreme subintervals of $\pi_{c}^{d}$.

(12.2) If $\boldsymbol{f}$ is a bounded function on $[a, b]$ and $[c, d] \subset[a, b]$ then for all $\pi_{0} \in \Pi_{a}^{b}$ there exists $\pi, \pi^{\prime}<\pi_{0}$ such that

$$
\begin{aligned}
& \left\|T \frown\left(f, \pi_{c}^{d}\right)\right\| \leqq \exp (2 M(b-a)) \cdot\|T \frown(f, \pi)\|, \\
& \left|S \frown\left(f, \pi_{c}^{d^{\prime}}\right)\right| \leqq \exp (2 M(b-a)) \cdot\left|S \frown\left(f, \pi^{\prime}\right)\right|,
\end{aligned}
$$

where $M=\sup _{a \leqq t \leqq b}|f(t)|$ and $\pi_{c}^{d}, \pi_{c}^{d^{\prime}}$ are the contractions of $\pi$ and $\pi^{\prime}$ to $[c, d]$.

Proof. Given $\pi_{0} \in \Pi_{a}^{b}$ we take $\pi<\pi_{0}$ such that $\left.{ }^{29}\right)|\pi| \leqq 1 / 2 M$. Let $\pi_{c}^{d}$ be the contraction of $\pi$ to $[c, d]$ and let $\sigma_{c}^{d}<\pi_{c}^{d}$ and $\pi_{c}^{d *}, \sigma_{c}^{d *}$ be valuations. Then there exists $\sigma<\pi$ of which $\sigma_{c}^{d}$ is the contraction but which coincides with $\pi$ outside $[c, d]$. Also there exist valuations $\pi^{*}, \sigma^{*}$ of which the above mentioned $\pi_{c}^{d *}, \sigma_{c}^{d *}$ are contractions but which coincide outside $[c, d]$. Applying the fundamental result (4.12) we get

$$
\begin{aligned}
\mid J \frown\left(f, \sigma, \sigma^{*}\right)- & J \frown\left(f, \pi, \pi^{*}\right) \mid \\
& \geqq \exp \left(-2 \sum_{\boldsymbol{\pi}-\pi_{c}^{d}}\left|f\left(t_{k}\right)\right|\left|\Delta_{k}\right|\right)\left|J \frown\left(f, \sigma_{c}^{d}, \sigma_{c}^{d *}\right)-J \frown\left(f, \pi_{c}^{d}, \pi_{c}^{d *}\right)\right| \\
& \geqq \exp (-2 M(b-a))\left|J \frown\left(f, \sigma_{c}^{d}, \sigma_{c}^{d *}\right)-J \frown\left(f, \pi_{c}^{d}, \pi_{c}^{d *}\right)\right| .
\end{aligned}
$$

Hence

$$
\|T \frown(f, \pi)\| \geqq \exp (-2 M(b-a))\left|J \frown\left(f, \sigma_{c}^{d}, \sigma_{c}^{d *}\right)-J \frown\left(f, \pi_{c}^{d}, \pi_{c}^{d *}\right)\right| .
$$

As this holds for all $\sigma_{c}^{d}<\pi_{c}^{d}$ and all valuations $\sigma_{c}^{d *}, \pi_{c}^{d *}$ we get

$$
\|T \frown(f, \pi)\| \geqq \exp (-2 M(b-a))\left\|T^{\frown}\left(f, \pi_{c}^{d}\right)\right\| .
$$

(b) is proved similarly. (Q.E.D.)

The corresponding result for $R$-integration is obvious. We leave the proof to the reader. Thus:

(12.3) If $[c, d] \subset[a, b]$ then for all $\pi \in \Pi_{a}^{b}$

$$
\left|S\left(f, \pi_{c}^{d}\right)\right| \leqq|S(f, \pi)|
$$

where $\pi_{c}^{d}$ is the contraction of $\pi$ to $[c, d]$.

Since if $[c, d] \subset[a, b]$ every partition of $[a, b]$ has a contraction to $[c, d]$ we get the desired result from Theorem 11.5, (12.2), and Lemma 10.4. Thus:

${ }^{(29)}$ We take a partition having this property so as to be able to apply the fundamental result (4.12). 
TheOREM 12.4. If $f$ is $R$-integrable on $[a, b]$ and $[c, d] \subset[a, b]$, then $f$ is $R$-integrable on $[c, d]$.

Also from (12.3) and Lemma 9.7 we get:

TheOREM 12.5. If $f$ is $R$-integrable on $[a, b]$ and $[c, d] \subset[a, b]$ then $f$ is $R$-integrable on $[c, d]$.

To turn to the question of the extension of the domain of integrability, suppose $a<c<b$. For all $\pi \in \Pi_{a}^{b}$ and all valuations $\pi^{*}$ there exist $\pi_{1} \in \Pi_{a}^{c}$, $\pi_{2} \in \Pi_{c}^{b}$ and valuations $\pi_{1}^{*}, \pi_{2}^{*}$ such that $\pi=\pi_{1} \cup \pi_{2}, \pi^{*}=\pi_{1}^{*} \cup \pi_{2}^{*}$. Hence

$$
\begin{aligned}
J \frown\left(f, \pi, \pi^{*}\right) & =J \frown\left(f, \pi_{1}, \pi_{1}^{*}\right) \cdot J \frown\left(f, \pi_{2}, \pi_{2}^{*}\right), \\
J\left(f, \pi, \pi^{*}\right) & =J\left(f, \pi_{1}, \pi_{1}^{*}\right)+J\left(f, \pi_{2}, \pi_{2}^{*}\right) .
\end{aligned}
$$

We are clearly led to the following result.

12.6. If $\left.{ }^{(30}\right) a<c<b$ then

$$
\begin{aligned}
\int_{a}^{b}(1+f d t) & =\int_{c}^{a}(1+f d t) \cdot \int_{c}^{b}(1+f d t), \\
\int_{a}^{b} f d t & =\int_{a}^{c} f d t+\int_{c}^{b} f d t .
\end{aligned}
$$

13. Uniform approach of $J\left(f, \pi_{x}^{y}, \pi_{x}^{y *}\right)$ to $\int_{x}^{y} f d t$.

DEFINITION 13.1. If $\pi \in \Pi_{a}^{b}$ and $\pi_{x}^{v}$ is the contraction of $\pi$ to $[x, y] \subset[a, b]$ then we shall say that $\lim _{\pi \downarrow} J\left(\pi_{x}^{v}, \pi_{x}^{y *}\right)=L(x, y)$ uniformly for $x, y \in[a, b]$ if and only if for all $\epsilon>0$ there exists $\pi_{\epsilon} \in \Pi_{a}^{b}$ such that, for all $x, y \in[a, b]$, $\pi_{x}^{y}<\left(\pi_{\epsilon}\right)_{x}^{y}$ implies for all valuations $\pi_{x}^{y *}$

$$
\left|J\left(\pi_{x}^{\nu}, \pi_{x}^{y *}\right)-L(x, y)\right|<\epsilon .
$$

In view of (12.3), (9.6) and Lemma 9.7 the following result may be obtained. We leave the proof to the reader.

(13.2) If $f$ is $R$-integrable on $[a, b]$ then

$$
\lim _{x \downarrow} J\left(f, \pi_{x}^{y}, \pi_{x}^{\nu *}\right)=\int_{x}^{y} f d t
$$

uniformly for $x, y \in[a, b]$.

14. Inverse of the product integral. The following result for the product integral corresponds to the result $\int_{a}^{b} f d t=-\int_{b}^{a} f d t$ for the additive case.

TheOREM 14.1( ${ }^{(31)}$. If $f$ is $R$-integrable on $[a, b]$ then:

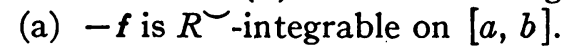

(30) The restriction that $a<c<b$ is only temporary. Cf. (14.4).

(21) A proof of this result for the case when $f$ is continuous has been given by Schlesinger $[1$, pp. 49-50]. 
(b) $\int_{a}^{b} \frown(1+f d t)$ has an inverse.

(c) $\left\{\int_{a}^{b} \backslash(1+f d t)\right\}^{-1}=\int_{a}^{b \smile}(1-f d t)$.

(a) will be shown in Chapter VI. It will turn up as a simple consequence of the connection we shall establish in the next chapter between additive and multiplicative integration ( $\left.{ }^{32}\right)$. (b) is a consequence of (3.2) and the following inequality (cf. (4.21))

$$
\left|\int_{a}^{b \frown}(1+f d t)-1\right| \leqq \exp (M(b-a))-1 .
$$

We shall leave the details of the argument to the reader. (c) follows from (4.6).

This theorem enables us to extend the notion of Riemann integral. According to Definitions 6.1 and 6.2 the expressions

$$
\int_{a}^{b}(1+f d t), \quad \int_{a}^{b \smile}(1+f d t), \quad \int_{a}^{b} f d t
$$

are not admissible in our system in case $a \geqq b$. Theorem 14.1 (c) suggests the definition 14.3 for the case $a>b$. (18.1) and (18.2) of the next chapter likewise suggest the following definition for the case $a=b$.

Definition 14.2. If $a=b$ then we define

$$
\int_{a}^{b}(1+f d t)=1=\int_{a}^{b \smile}(1+f d t), \quad \int_{a}^{b} f d t=0 .
$$

Definition 14.3. If $a>b$ then we define

(i)

$$
\begin{aligned}
\int_{a}^{b}(1+f d t) & =\int_{b}^{a \smile}(1-f d t), \\
\int_{a}^{b \smile}(1+f d t) & =\int_{b}^{a}(1-f d t), \\
\int_{a}^{b} f d t & =-\int_{b}^{a} f d t .
\end{aligned}
$$

As a corollary of these definitions we obtain:

(14.4). The restriction that $a<c<b$ in Lemma 12.6 can be removed.

V. Equivalence OF ADDITIVE AND MULTIPLICATIVE INTEGRABILITY

Our main purpose in this chapter is to establish the equivalence of additive and multiplicative Riemann integrability of functions on $[a, b]$ into a normed ring $X$. In the following section we obtain the lemmas needed for this purpose.

(2) Cf. Theorem 16.3 and (19.1). 
15. Preliminary remarks. Suppose $\pi_{0}=\left\{\Delta_{1}, \cdots, \Delta_{r}\right\} \in \Pi_{a}^{b}$ and that $\pi_{k} \in \Pi_{\Delta_{k}}$. It is clear that $\pi=\pi_{1} \cup \ldots \cup \pi_{r} \in \Pi_{a}^{b}$ and that if $\pi^{\prime}<\pi$ then $\pi^{\prime}$ is of the type $\pi^{\prime}=\pi_{1}^{\prime} \cup \ldots \cup \pi_{r}^{\prime}$ where $\pi_{k}^{\prime}<\pi_{k}$. Also all valuations $\pi^{*}, \pi^{\prime *}$ can be written $\pi^{*}=\pi_{1}^{*} \cup \ldots \cup \pi_{r}^{*}, \pi^{\prime *}=\pi_{1}^{\prime *} \cup \ldots \cup \pi_{r}^{\prime *}$ where $\pi_{k}^{*}, \pi_{k}^{\prime *}$ are valuations of $\pi_{k}, \pi_{k}^{\prime}$ respectively. Hence

$$
\begin{gathered}
J \frown\left(f, \pi, \pi^{*}\right)=\prod_{k=1}^{r} J \frown\left(f, \pi_{k}, \pi_{k}^{*}\right), \\
J \frown\left(f, \pi^{\prime}, \pi^{* *}\right)=\prod_{k=1}^{r} J \frown\left(f, \pi_{k}^{\prime}, \pi_{k}^{\prime *}\right) .
\end{gathered}
$$

Suppose next that $f$ is a bounded function on $[a, b]$ and that $M=\sup _{a \leq t \leq b}|f(t)|$. Applying (4.42) we have

$$
\begin{aligned}
& \left|J \frown\left(f, \pi, \pi^{*}\right)-J \frown\left(f, \pi^{\prime}, \pi^{\prime *}\right)\right| \\
& \leqq \sum_{k=1}^{r}\left[\left|\prod_{i=1}^{k-1} J \frown\left(f, \pi_{i}, \pi_{i}^{*}\right)\right| \cdot\left|\prod_{i=k+1}^{r} J \frown\left(f, \pi_{i}^{\prime}, \pi_{i}^{\prime *}\right)\right|\right. \\
& \left.\cdot\left|J \frown\left(f, \pi_{k}, \pi_{k}^{*}\right)-J \frown\left(f, \pi_{k}^{\prime}, \pi_{k}^{\prime *}\right)\right|\right]
\end{aligned}
$$

or from (4.22)

$$
\begin{aligned}
\mid J \frown\left(f, \pi, \pi^{*}\right)-J \frown & \left(f, \pi^{\prime}, \pi^{\prime *}\right) \mid \\
& \leqq \exp (2 M(b-a)) \cdot \sum_{k=1}^{r}\left|J \frown\left(f, \pi_{k}, \pi_{k}^{*}\right)-J \frown\left(f, \pi_{k}^{\prime}, \pi_{k}^{\prime *}\right)\right| \\
& \leqq \exp (2 M(b-a)) \cdot \sum_{k=1}^{r}\left\|T^{\frown}\left(f, \pi_{k}\right)\right\| .
\end{aligned}
$$

Hence

$$
\left\|T^{\sim}(f, \pi)\right\| \leqq \exp (2 M(b-a)) \cdot \sum_{k=1}^{r}\left\|T^{\frown}\left(f, \pi_{k}\right)\right\| .
$$

Thus:

(15.1) If $f$ is a bounded function on $[a, b], M=\sup _{a \leqq t \leqq b}|f(t)|$ and $\pi_{0}=\left\{\Delta_{1}, \cdots, \Delta_{r}\right\} \in \Pi_{a}^{b}$ then $\pi_{k} \in \Pi_{\Delta_{k}}$ implies

$$
\|T \frown(f, \pi)\| \leqq \exp (2 M(b-a)) \cdot \sum_{k=1}^{r}\left\|T \frown\left(f, \pi_{k}\right)\right\| .
$$

In a similar way it is even easier to prove the corresponding result for $R$-integration. Thus:

(15.2) If $f$ is any function on $[a, b]$ and $\pi_{0}=\left\{\Delta_{1}, \cdots, \Delta_{r}\right\} \in \Pi_{a}^{b}$ then 
$\pi_{k} \in \Pi_{\Delta_{k}}$ implies

$$
|S(f, \pi)| \leqq \sum_{k=1}^{r}\left|S\left(f, \pi_{k}\right)\right|
$$

The next result deals with the order of closeness of the approximative sum and product. Suppose $f$ is any bounded function on $[a, b]$ and $\Delta$ is a subinterval of $[a, b]$. Let $\pi_{\Delta} \in \Pi_{\Delta}$ and $\pi_{\Delta}^{*}$ be a valuation of $\pi_{\Delta}$. Then from (4.32)

$$
\begin{aligned}
\mid J \sim\left(f, \pi_{\Delta}, \pi_{\Delta}^{*}\right)-\{1+ & \left.J\left(f, \pi_{\Delta}, \pi_{\Delta}^{*}\right)\right\} \mid \\
& =\left|\prod_{\alpha}\left\{1+f\left(t_{k}^{\alpha}\right)\left|\Delta_{k}^{\alpha}\right|\right\}-\left\{1+\sum_{\alpha} f\left(t_{k}^{\alpha}\right)\left|\Delta_{k}^{\alpha}\right|\right\}\right| \\
& \leqq(\exp (M|\Delta|)-1) M|\Delta|
\end{aligned}
$$

where $M=\sup _{a \leq t \leq b}|f(t)|$.

Thus as $|\Delta| \rightarrow 0$, the difference on the L.H.S. is an infinitesimal of higher order than $|\Delta|$. The whole demonstration of the equivalence of $R$ - and $R$-integrability hinges on this result. Thus:

(15.3) If $f$ is a bounded function on $[a, b]$ then for all $\epsilon>0$ there exists $\delta_{\epsilon}>0$ such that $\Delta \subset[a, b]$ and $|\Delta|<\delta_{\epsilon}$ implies for all $\pi_{\Delta} \in \Pi_{\Delta}$ and all valuations $\pi_{\Delta}^{*}$

$$
\left|J \frown\left(f, \pi_{\Delta}, \pi_{\Delta}^{*}\right)-\left\{1+J\left(f, \pi_{\Delta}, \pi_{\Delta}^{*}\right)\right\}\right|<\epsilon|\Delta| .
$$

16. Equivalence of $R$-and $R$-integrability. To establish the equivalence of $R \frown$ - and $R$-integrability it will suffice (cf. Lemma 9.7,10.1, and 10.4) to establish the following deductive chain, arrows indicating implication,

$$
\lim _{\pi_{0} \downarrow} \inf _{\pi<\pi_{0}}|S \frown(f, \pi)|=0 \rightarrow \inf _{\pi}|S(f, \pi)|=0 \rightarrow \inf _{\pi}\|T \frown(f, \pi)\|=0 .
$$

In so doing we shall also be showing the sufficiency of the condition $\lim _{\pi_{0} \downarrow} \inf _{\pi<\pi_{0}}|S \sim(f, \pi)|=0$ for $R \frown$-integrability.

LEMMA 16.1. $\lim _{\pi_{0} \downarrow} \inf _{\pi} \prec \pi_{0}|S \sim(f, \pi)|=0$ implies inf $\pi|S(f, \pi)|=0$.

Proof. Suppose $\lim _{\pi_{0} \downarrow} \inf _{\pi}<\pi_{0}|S \sim(f, \pi)|=0$. Then from (11.4) we know that $f$ is bounded. Appealing to (15.3) we find that given any $\epsilon>0$ there exists $\delta_{\epsilon}>0$ such that $\pi_{0}=\left\{\Delta_{1}, \cdots, \Delta_{r}\right\} \in \Pi_{a}^{b}$ and $\left|\pi_{0}\right|<\delta_{\epsilon}$ implies for all $\pi_{k} \in \Pi_{\Delta_{k}}$ and all valuations $\pi_{k}^{*}$

$$
\left|J \frown\left(f, \pi_{k}, \pi_{k}^{*}\right)-\left\{1+J\left(f, \pi_{k}, \pi_{k}^{*}\right)\right\}\right| \leqq \epsilon\left|\Delta_{k}\right| / 3(b-a) .
$$

From $(12.2(\mathrm{~b}))$ we also know that $\lim _{\pi_{k}^{0} \downarrow} \inf _{\pi}\left\langle\pi_{k}^{0}\left|S \sim\left(f, \pi_{k}\right)\right|=0\right.$. Therefore there exists $\pi_{k} \in \Pi_{\Delta_{k}}$ such that

$$
\left|S \frown\left(f, \pi_{k}\right)\right| \leqq \epsilon\left|\Delta_{k}\right| / 3(b-a) .
$$


For this $\pi_{k}$ and all its valuations $\pi_{k}^{*}, \pi_{k}^{\dagger}$ we have

$$
\begin{aligned}
\left|J\left(f, \pi_{k}, \pi_{k}^{*}\right)-J\left(f, \pi_{k}, \pi_{k}^{\dagger}\right)\right| \leqq & \left|1+J\left(f, \pi_{k}, \pi_{k}^{*}\right)-J \frown\left(f, \pi_{k}, \pi_{k}^{*}\right)\right| \\
& +\left|J \frown\left(f, \pi_{k}, \pi_{k}^{*}\right)-J \frown\left(f, \pi_{k}, \pi_{k}^{\dagger}\right)\right| \\
& +\left|J \frown\left(f, \pi_{k}, \pi_{k}^{\dagger}\right)-\left\{1+J\left(f, \pi_{k}, \pi_{k}^{\dagger}\right)\right\}\right| \\
\leqq & \epsilon \Delta_{k} \mid /(b-a)
\end{aligned}
$$

from (1) and (2). Hence $\left|S\left(f, \pi_{k}\right)\right| \leqq \epsilon\left|\Delta_{k}\right| /(b-a)$. If we let $\pi=\pi_{1} \cup \cdots \cup \pi_{r}$ it follows from (15.2) that

$$
|S(t, \pi)| \leqq \sum_{k=1}^{r} \epsilon\left|\Delta_{k}\right| /(b-a)=\epsilon
$$

and the proof is completed. (Q.E.D.)

Lemma 16.2. $\inf _{x}|S(f, \pi)|=0$ implies $\inf _{x}\left\|T^{\sim}(f, \pi)\right\|=0$.

Proof. Suppose $\inf _{\pi}|S(f, \pi)|=0$. Then, by Lemma 9.7, $f$ is $R$-integrable and therefore (cf. (11.6)) bounded. From here the proof develops in a similar way to that of the last lemma. We appeal to (15.3), (12.2(a)) and finally to (15.1). We leave it to the reader to supply the details. (Q.E.D.)

As remarked above we can summarise the results obtained in this section in the following two theorems.

THEOREM 16.3. $f$ is $R$-integrable if and only if $f$ is $R$-integrable.

THEOREM 16.4. $f$ is $R$-integrable if and only if $\lim _{\pi_{0} \downarrow} \inf _{x}<\pi_{0}|S(f, \pi)|=0$.

Theorem 16.3 provides us with a powerful weapon with which to attack problems of product integrability.

17. Continuity almost everywhere. L. Graves [1, pp. 168-169, Theorem 1] has shown that every function continuous almost everywhere $\left({ }^{33}\right)$ and bounded is $R$-integrable. That continuity a.e. is not in general a necessary condition for $R$-integrability has again been shown by Graves $[1, p .166]$. We shall now show that when the normed ring $X$ is finite-dimensional, continuity a.e. does constitute a necessary condition for $R$-integrability.

If $X$ is finite-dimensional there exist basis elements $\alpha_{1}, \cdots, \alpha_{r} \in X$ such that every element $x \in X$ is a linear combination of these $x=c_{1} \alpha_{1}+\cdots+c_{r} \alpha_{r}$ where each $c_{i}$ is real( $\left.{ }^{34}\right)$. If now $f$ be a function on $[a, b]$ into $X$, we have for each $t \in[a, b]$

$$
f(t)=g_{1}(t) \alpha_{1}+\cdots+g_{r}(t) \alpha_{r}
$$

where each function $g_{i}(t)$ is real. Hence

(28) That is, one whose points of discontinuity form a set of measure zero.

(M) This holds even if the ring has complex scalers. 


$$
J\left(f, \pi, \pi^{*}\right)=\sum_{k=1}^{n(\pi)}\left(\sum_{i=1}^{r} g_{i}\left(t_{k}\right) \boldsymbol{\alpha}_{i}\right)\left|\Delta_{k}\right|=\sum_{i=1}^{r} J\left(g_{i}, \pi, \pi^{*}\right) \boldsymbol{\alpha}_{i} .
$$

Suppose now that $f$ is $R$-integrable on $[a, b]$. Then $J\left(f, \pi, \pi^{*}\right)$ and therefore (since the $\alpha_{i}$ are linearly independent) each $J\left(g_{i}, \pi, \pi^{*}\right)$ converges as $\pi \downarrow$. But since each $g_{i}$ is real this implies that each $g_{i}$ is continuous a.e. on $[a, b]$. From (1) it follows that $f$ is continuous a.e. on $[a, b]$. We therefore have the following theorem.

THEOREM 17.1. Boundedness and continuity a.e. constitute always a suffcient condition for $R$-integrability. When the normed ring $X$ is finite-dimensional they constitute also a necessary condition.

18. Absolute continuity of the indefinite integrals. We shall recall here for subsequent use two known results (see L. M. Graves [1, pp. 170, Theorem 3] and L. Schlesinger $[1$, p. 45]).

(18.1) If $F(x)=\int_{a}^{x} f d t, a \leqq x \leqq b$, and $M=\sup _{a \leqq t \leqq b}|f(t)|$ then for all $x$, $y \in[a, b]$

$$
|F(x)-F(y)| \leqq M|x-y|
$$

and $F$ is absolutely continuous and hence of bounded variation.

(18.2) If $G(x)=\int_{a}^{x}(1+f d t), a \leqq x \leqq b$, and $M=\sup _{a \leqq i \leqq b}|f(t)|$, then for all $x, y \in[a, b]$

$$
|G(x)-G(y)| \leqq \exp (M(b-a)) \cdot[\exp (M(b-a))+\operatorname{Osc}(f,[a, b])] \cdot|x-y|
$$

and $G$ is absolutely continuous and hence of bounded variation.

\section{Structure of the Class of $R$-Integrable functions}

19. Structure of the class $R$. We shall now determine the topological and algebraic structure of the class $R$ of all $R$-integrable functions on a real interval into a normed ring $X$. On the algebraic side we have the following results.

(19.1) If $f$ is $R$-integrable on $[a, b]$ and if $c \in X$ or $c$ is a complex number, then so is $c f$ and

$$
\int_{a}^{b} c f d t=c \int_{a}^{b} f d t .
$$

(19.2) If $\boldsymbol{f}$ and $\boldsymbol{\phi}$ are $R$-integrable on $[a, b]$ so is $\boldsymbol{f}+\boldsymbol{\phi}$ and

$$
\int_{a}^{b}(f+\phi) d t=\int_{a}^{b} f d t+\int_{a}^{b} \phi d t
$$

The question as to whether $R$ is closed under ring multiplication is best postponed until after a study of the topological structure of $R$. On the topo- 
logical side we have the following results which can be obtained by essentially classical methods.

(19.3) If each function $f_{n}$ is $R$-integrable on $[a, b]$ and $f_{n} \rightarrow f$ uniformly on $[a, b]$ then $f$ is $R$-integrable on $[a, b]$ and

$$
\int_{a}^{b} f d t=\lim _{n \rightarrow \infty} \int_{a}^{b} f_{n} d t .
$$

(19.4) If (i) each function $f_{n}$ is differentiable on $[a, b]$, (ii) $f_{n} \rightarrow f$ on $[a, b]$, (iii) the sequence $\left.{ }^{35}\right)\left(f_{n}^{*}\right)_{n=1}^{\infty}$ converges uniformly on $[a, b]$, then $f$ is differentiable on $[a, b]$ and

$$
f^{*}=\lim _{n \rightarrow \infty} f_{n}^{\cdot} .
$$

The following result however requires proof.

(19.5) If each function $f_{n}$ is $R$-integrable on $[a, b]$ and $f_{n} \rightarrow f$ uniformly on $[a, b]$ then

$$
\int_{a}^{b}(1+f d t)=\lim _{n \rightarrow \infty} \int_{a}^{b r}\left(1+f_{n} d t\right)
$$

Proof. The existence of $\int_{a}^{b}(1+f d t)$ follows from (19.3). Now for all partitions $\pi$ and all valuations $\pi^{*}$ we have from (4.44)

$$
\begin{aligned}
\mid J \frown\left(f_{m}, \pi, \pi^{*}\right)- & J \frown\left(f, \pi, \pi^{*}\right) \mid \\
& \leqq \exp (M(b-a)) \cdot\left\{\exp \left(\sum_{k=1}^{n(\pi)}\left|f_{m}\left(t_{k}\right)-f\left(t_{k}\right)\right|\left|\Delta_{k}\right|\right)-1\right\} .
\end{aligned}
$$

where $M=\sup _{a \leqq t \leq b}|f(t)|$. Since $f_{n} \rightarrow f$ uniformly on $[a, b]$, for every $\epsilon>0$ there exists a positive integer $m_{\epsilon}$ such that $m \geqq m_{\mathrm{e}}$ implies for all $t \in[a, b]$

$$
\left|f(t)-f_{m}(t)\right|<\frac{1}{b-a} \operatorname{Ln}\{1+\epsilon \exp (-M(b-a))\} .
$$

Thus there exists $m_{\epsilon}$ such that $m \geqq m_{\epsilon}$ implies for all $\pi, \pi^{*}$

$$
\begin{aligned}
& \left|J \frown\left(f_{m}, \pi, \pi^{*}\right)-J \frown\left(f, \pi, \pi^{*}\right)\right| \\
& <\exp (M(b-a)) \cdot\left\{\exp \left(\frac{1}{b-a} \operatorname{Ln}\{1+\exp (-M(b-a)) \epsilon\} \sum_{k=1}^{n(\pi)}\left|\Delta_{k}\right|\right)-1\right\} \\
& <\epsilon .
\end{aligned}
$$

Since this holds for all $\pi, \pi^{*}$ we have

$$
\left|\int_{a}^{b}\left(1+f_{m} d t\right)-\int_{a}^{b}(1+f d t)\right|<\epsilon, \quad m \geqq m_{e},
$$

(अ) $f_{n} \cdot$ denotes the ordinary additive derivative of $f_{n}$. 
and the proof is completed. (Q.E.D.)

We may summarize these results in the following two theorems.

THeOREM 19.6. The class $\boldsymbol{R}$ of all $R$-integrable functions on $[a, b]$ into $\boldsymbol{X}$ is a Banach space which is a (closed additive) subgroup of the normed ring of all bounded functions on $[a, b]$ into $\boldsymbol{X}$ under the uniform topology $|\boldsymbol{f}-\boldsymbol{\phi}|$ $=\sup _{a \leqq t \leqq b}|f(t)-\phi(t)|$.

THEOREM 19.7. Indefinite $R-$ and $R$-integration are continuous operations on the class $R$ under the uniform topology. Indefinite $R$-integration is also a linear operation.

20. Closure under ring multiplication. $R$ is certainly closed under ring multiplication when the normed ring $X$ is finite-dimensional. For we know (cf. Theorem 17.1) that, in the finite-dimensional case, boundedness and continuity almost everywhere constitute both a necessary and sufficient condition for $R$-integrability. For the finite-dimensional case we may therefore strengthen Theorem 19.6 as follows.

TheOREM 20.1. The class $R$ of all $R$-integrable functions on $[a, b]$ into $a$ finite-dimensional normed ring $X$ is itself a normed ring which is a (closed) subring of the normed ring of all bounded functions on $[a, b]$ into $X$ under the uniform topology $|\boldsymbol{f}-\boldsymbol{\phi}|=\sup _{a \leqq t \leqq b}|\boldsymbol{f}(t)-\boldsymbol{\phi}(t)|$.

The following counter-example due to Garrett Birkhoff shows that in general $R$ is not closed under ring multiplication.

EXAMPLE 20.2. Let $X$ be the class of all bounded (continuous) linear transformations on the space $B$ of all real $R$-integrable functions on $[0,1]$. From Theorem 19.6 we know that $B$ is a Banach space under the norm $|f|$ $=\sup _{0 \leqq t \leq 1}|f(t)|$. It follows (cf. Banach $[1$, p. 54]) that $X$ is a normed ring, the norm $|T|$ of a bounded linear transformation $T \in X$ being given by

$$
|T|=\sup _{f \in B, f \neq 0} \frac{|T(f)|}{|f|} .
$$

For all $t \in[0,1]$ we let $T_{t}, U_{t}$ be such that for all $f \in B$ and $0 \leqq x \leqq 1$

$$
\begin{aligned}
\left(T_{t} f\right) x & =\left\{\begin{array}{lr}
f(0) & \text { if } t \text { is irrational and } x=t, \\
0 & \text { otherwise, }
\end{array}\right. \\
\left(U_{t} f\right) x & = \begin{cases}f(t) & \text { if } x=0, \\
0 & \text { if } x=0 .\end{cases}
\end{aligned}
$$

It is easy to see that, for each $t \in[0,1], T_{t}, U_{t} \in X\left({ }^{36}\right)$. It may be shown that the functions $T_{t}, U_{t}$ are $R$-integrable on $[0,1]$ and that

(36) That is, for each $t, U_{t}, T_{t}$ are bounded linear transformations on $B$. 


$$
\int_{0}^{1} T_{t} d t=\Theta, \quad \int_{0}^{1} U_{t} d t=\Im
$$

where $\Theta$ is the zero-element $\left.{ }^{(37}\right)$ of $X$, and $J$ is the linear transformation carrying each $f \in B$ into the function $\phi$ such that $\phi(x)=0$ if $x \neq 0, \phi(0)=\int_{0}^{1} f d t\left({ }^{38}\right)$.

But the function $U_{t} \cdot T_{t}$ is not $R$-integrable. For let $f \in B$ and $t \in[0,1]$; then

$$
\left(U_{t} T_{t} f\right) 0=\left\{\begin{array}{l}
f(0) \\
0
\end{array}\right.
$$

if $t$ is irrational,

if $t$ is rational.

If for all $\pi \in \Pi_{n}^{1}$ we let $\pi^{*}, \pi^{\dagger}$ be valuations of $\pi$ consisting only of rational and only of irrational points respectively, then

$$
\sum_{\pi, \pi^{*}}\left|\Delta_{k}\right|\left(U_{t_{k}} T_{t_{k}} f\right) 0=0, \quad \sum_{\pi, \pi^{\dagger}}\left|\Delta_{k}\right|\left(U_{t_{k}} T_{t_{k}} f\right) 0=f(0) \sum\left|\Delta_{k}\right|=f(0) .
$$

Hence for all $\pi\left|S\left(U_{t} \cdot T_{t}, \pi\right)\right| \geqq|f(0)| /|f|$. It follows that $U_{t} \cdot T_{t}$ is not $R$-integrable on $[0,1]$.

It is easily seen however that $T_{t} \cdot U_{t}$ is $R$-integrable. For

$$
\left(T, U_{t} f\right) x=\left\{\begin{array}{lr}
f(t) & \text { if } t \text { is irrational and } x=t, \\
0 & \text { otherwise. }
\end{array}\right.
$$

If now $\pi \in \Pi_{0}^{1}$ and $\pi^{*}$ be any valuation of $\pi$, then each $x \in[0,1]$ belongs to some $\Delta_{i_{x}} \in \pi$. Hence

$$
\sum_{x, x^{*}}\left|\Delta_{k}\right|\left(T_{t_{k}} U_{t_{k}} f\right) x= \begin{cases}f\left(t_{i_{x}}\right)\left|\Delta_{i_{x}}\right| & \text { if } t_{i_{x}} \text { is irrational and } x=t_{i_{x}}, \\ 0 & \text { otherwise. }\end{cases}
$$

Since $\left|\Delta_{i_{x}}\right| \rightarrow 0$ as $\pi \downarrow$, it follows that $T_{t} \cdot U_{t}$ is $R$-integrable on $[0,1]$ and that $\int_{0}^{1} T_{t} U_{t} d t=\Theta$.

We thus see that when $X$ is infinite-dimensional the $R$-integrability of $f$ and $\phi$ does not in general imply that of $f \phi$. Nor does the $R$-integrability of $f \phi$ imply that of $\phi f$.

\section{THE PEANO SERIES}

In this chapter we shall establish the validity of the Peano series:

$$
\begin{aligned}
\int_{a}^{b}(1+f d t)=1 & +\int_{a}^{b} f d t+\int_{a}^{b} \int_{a}^{t_{2}} f\left(t_{1}\right) f\left(t_{2}\right) d t_{1} d t_{2} \\
& +\int_{a}^{b} \int_{a}^{t_{3}} \int_{a}^{t_{2}} f\left(t_{1}\right) f\left(t_{2}\right) f\left(t_{3}\right) d t_{1} d t_{2} d t_{3}+\cdots
\end{aligned}
$$

${ }^{(37)} \Theta$ is the linear transformation carrying each $f \in B$ into the zero-function.

(38) The new function $\phi$ has a single discontinuity and therefore belongs to $B$. 
for any $R$-integrable function $f$. We shall then show the validity of the integral equation mentioned in $\$ 2\left({ }^{39}\right)$ for all $R$-integrable functions. Finally we shall see what simplifications result when the normed ring $X$ is commutative.

In view of Example 20.2 it is clear that we must first establish the existence of the iterated integrals occurring in the Peano series for any $R$-integrable function.

21. Iterated additive integration. We shall actually establish the stronger result that the functions

$$
\left\{\int_{a}^{x} f d t\right\} \phi(x), \quad f(x) \int_{a}^{x} \phi d t \quad(a \leqq x \leqq b)
$$

are $R$-integrable on $[a, b]$ whenever $f$ and $\phi$ are. The proof of this rests on the following results, the proof of the first of which is obvious.

$$
\sum_{k=1}^{n}\left[\left(\sum_{i=1}^{k-1} \mathbf{x}_{i}\right)\left(y_{k}-z_{k}\right)\right]=\sum_{k=1}^{n-1}\left[x_{k} \sum_{i=k+1}^{n}\left(y_{i}-z_{i}\right)\right] \text {. }
$$

(21.2) If $f$ is $R$-integrable on $[a, b]$ and $\phi$ is bounded then

$$
\lim _{-\downarrow}\left|\sum_{k=1}^{n(x)} \sum_{i=1}^{k-1} f\left(t_{i}\right)\right| \Delta_{i}\left|\cdot \phi\left(t_{k}\right)\right| \Delta_{k}\left|-\sum_{k=1}^{n(x)}\left\{\int_{a}^{t_{k}} f\left(t_{i}\right) d t_{i}\right\} \phi\left(t_{k}\right)\right| \Delta_{k}||=0 .
$$

Proof. Denoting the absolute difference on the L.H.S. by $D\left(\pi, \pi^{*}\right)$ we first observe that

$$
D\left(\pi, \pi^{*}\right) \leqq M_{1} \sum_{k=1}^{n(\pi)}\left|\sum_{i=1}^{k-1} f\left(t_{i}\right)\right| \Delta_{i}\left|-\int_{a}^{t_{k}} f\left(t_{i}\right) d t_{i}\right|\left|\Delta_{k}\right|
$$

where $M_{1}=\sup _{a \leq t \leq b}|\phi(t)|$. For all $\pi$ and $\pi^{*}$ let us define $g\left(\pi, \pi^{*}, x\right)$ as the positive step-function on $[a, b]$ such that for all $x \in \Delta_{k} \in \pi$

$$
g\left(\pi, \pi^{*}, x\right)=\left|\sum_{i=1}^{k-1} f\left(t_{i}\right)\right| \Delta_{i}\left|-\int_{a}^{t_{k}} f\left(t_{i}\right) d t_{i}\right| .
$$

Then

$$
g\left(\pi, \pi^{*}, x\right) \leqq 2 M_{2}(b-a)
$$

where $M_{2}=\sup _{a \leq t \leq b}|f(t)|$. Also for a given $x \in[a, b]$ let $\Delta_{k(x)}=\left[x_{k(\pi)-1}, x_{k(\pi)}\right]$ be the subinterval of $\pi$ containing $x$. Then

$$
\begin{aligned}
g\left(\pi, \pi^{*}, x\right) \leqq & \left|\sum_{i=1}^{k(\pi)-1} f\left(t_{i}\right)\right| \Delta_{i}\left|-\int_{a}^{x_{k(\pi)-1}} f\left(t_{i}\right) d t_{i}\right| \\
& +\left|\int_{a}^{x_{k(\pi)-1}} f\left(t_{i}\right) d t_{i}-\int_{a}^{t_{k(\pi)}} f\left(t_{i}\right) d t_{i}\right| .
\end{aligned}
$$

(39) Cf. equation (5) of that section. 
Since, as $\pi \downarrow, x_{k(\pi)-1}, x_{k(\pi)}$ and therefore $t_{k(x)}$ approach $x$, it follows from (13.2) and (18.1) that $\lim _{\pi \downarrow} g\left(\pi, \pi^{*}, x\right)=0$. Hence, applying Lemma A1.2 (Appendix 1), we get

$$
\lim _{x \downarrow} \sum_{k=1}^{n(\pi)}\left|\sum_{i=1}^{k-1} f\left(t_{i}\right)\right| \Delta_{i}\left|-\int_{0}^{t_{k}} f\left(t_{i}\right) d t_{i}\right|\left|\Delta_{k}\right|=0 .
$$

The result now follows from (1). (Q.E.D.)

THEOREM 21.3. If $f$ and $\phi$ are $R$-integrable on $[a, b]$ then so are $\left\{\int_{a}^{x} f d t\right\} \phi(x)$ and $\phi(x) \int_{a}^{x} f d t(a \leqq x \leqq b)$.

Proof. Let $F(x)=\left\{\int_{a}^{x} f d t\right\} \phi(x), a \leqq x \leqq b$. From (21.2) we know that given any $\epsilon>0$ there exists $\pi_{0} \in \Pi_{a}^{b}$ such that for all $\pi<\pi_{0}$ and all valuations $\pi^{*}$ we have

$$
\left|\sum_{k=1}^{n(\pi)}\left(\int_{a}^{t_{k}} f d t\right) \phi\left(t_{k}\right)\right| \Delta_{k}\left|-\sum_{k=1}^{n(\pi)}\left\{\sum_{i=1}^{k-1} f\left(t_{i}\right)\left|\Delta_{i}\right|\right\} \phi\left(t_{k}\right)\right| \Delta_{k}||<\epsilon / 4,
$$

and therefore with an obvious notation

$$
\begin{aligned}
\left|J\left(F, \pi, \pi^{*}\right)-J\left(F, \pi, \pi^{\dagger}\right)\right| \leqq & \left|\sum_{k=1}^{n(\pi)}\left\{\sum_{i=1}^{k-1} f\left(t_{i}\right)\left|\Delta_{i}\right|\right\} \phi\left(t_{k}\right)\right| \Delta_{k} \mid \\
& -\sum_{k=1}^{n(\pi)}\left\{\sum_{i=1}^{k-1} f\left(t_{i}^{\prime}\right)\left|\Delta_{i}\right|\right\} \phi\left(t_{k}^{\prime}\right)\left|\Delta_{k}\right| \mid+\epsilon / 2 .
\end{aligned}
$$

Now the first term on the R.H.S. of (1) is not greater than

$$
\begin{aligned}
\mid \sum_{k=1}^{n(\pi)}\left\{\sum_{i=1}^{k-1} f\left(t_{i}\right)\left|\Delta_{i}\right|\right. & \left.-\sum_{i=1}^{k-1} f\left(t_{i}^{\prime}\right)\left|\Delta_{i}\right|\right\} \phi\left(t_{k}\right)\left|\Delta_{k}\right| \mid \\
& +\left|\sum_{k=1}^{n(\pi)}\left(\sum_{i=1}^{k-1} f\left(t_{i}\right)\left|\Delta_{i}\right|\right)\left\{\phi\left(t_{k}\right)-\phi\left(t_{k}^{\prime}\right)\right\}\right| \Delta_{k}|| .
\end{aligned}
$$

The first term of (2) is in turn not greater than

$$
\sum_{k=1}^{n(\pi)}\left|S\left(f, \pi_{a}^{k-1}\right)\right| M_{\phi}\left|\Delta_{k}\right| \leqq|S(f, \pi)| M_{\phi}(b-a)
$$

from (12.3), $\pi_{a}^{k-1}$ being the contraction of $\pi$ to $\left[a, x_{k-1}\right]$ and $M_{\phi}=\sup _{a \leq t \leq b}|\phi(t)|$. And from (21.1) the second term of (2) equals

$$
\begin{aligned}
\mid \sum_{k=1}^{n(x)-1}\left[f ( t _ { k } ) | \Delta _ { k } | \cdot \sum _ { i = k + 1 } ^ { n ( \pi ) } \left\{\phi\left(t_{i}\right)\right.\right. & \left.\left.-\phi\left(t_{i}^{\prime}\right)\right\}\left|\Delta_{i}\right|\right] \\
& \leqq \sum_{k=1}^{n(x)} M_{f}\left|\Delta_{k}\right|\left|S\left(\phi, \pi_{k}^{n}\right)\right| \leqq|S(\phi, \pi)| M_{f}(b-a)
\end{aligned}
$$


again from (12.3), $\pi_{k}^{n}$ being the contraction of $\pi$ to $\left[x_{k}, b\right]$ and $M_{f}$ $=\sup _{a \leq t \leq b}|f(t)|$. (1) therefore becomes

$$
\left|J\left(F, \pi, \pi^{*}\right)-J\left(F, \pi, \pi^{z}\right)\right| \leqq\left\{M_{\phi}|S(f, \pi)|+M_{f}|S(\phi, \pi)|\right\}(b-a)+\epsilon / 2 .
$$

Hence $|S(F, \pi)| \leqq\left\{M_{\phi}|S(f, \pi)|+M_{f}|S(\phi, \pi)|\right\}(b-a)+\epsilon / 2$. Since $f$ and $\phi$ are $R$-integrable it clearly follows from this inequality that so is $F$. The $R$-integrability of $\phi(x) \int_{a}^{x} f d t$ follows from the duality principle. (Q.E.D.)

As corollaries of this result we at once obtain the following extensions for iterated integration of higher degree.

(21.4) If $\boldsymbol{f}$ is $R$-integrable on $[a, b]$ then so is the function

$$
\left\{\int_{a}^{x} \int_{a}^{t_{r}} \cdots \int_{a}^{t_{2}} f\left(t_{1}\right) \cdots f\left(t_{r}\right) d t_{1} \cdots d t_{r}\right\} f(x) \quad(a \leqq x \leqq b)
$$

for all positive integers $r$.

(21.5) If $f$ is $R$-integrable on $[a, b]$ then for all positive integers $r$

$$
\begin{aligned}
\lim _{\pi \downarrow} \sum_{k_{r}=1}^{n(\pi)} \sum_{k_{r-1}=1}^{k_{r}-1} \cdots \sum_{k_{1}=1}^{k_{2}-1} f\left(t_{k_{1}}\right)\left|\Delta_{k_{1}}\right| & \cdots f\left(t_{k_{r}}\right)\left|\Delta_{k_{r}}\right| \\
& =\int_{a}^{b} \int_{a}^{t_{r}} \cdots \int_{a}^{t_{2}} f\left(t_{1}\right) \cdots f\left(t_{r}\right) d t_{1} \cdots d t_{r} .
\end{aligned}
$$

22. The Peano series.

THEOREM 22.1. If $f$ is $R$-integrable on $[a, b]$ then

$$
\begin{aligned}
\int_{a}^{b}(1+f d t)=1 & +\int_{a}^{b} f d t+\int_{a}^{b} \int_{a}^{t_{2}} f\left(t_{1}\right) f\left(t_{2}\right) d t_{1} d t_{2} \\
& +\int_{a}^{b} \int_{a}^{t_{3}} \int_{a}^{t_{2}} f\left(t_{1}\right) f\left(t_{2}\right) f\left(t_{3}\right) d t_{1} d t_{2} d t_{3}+\cdots,
\end{aligned}
$$

the series on the R.H.S. being convergent.

Proof. For brevity we shall write $x_{k}$ for $f\left(t_{k}\right)\left|\Delta_{k}\right|$. Then

$$
\begin{aligned}
J \frown\left(f, \pi, \pi^{*}\right)= & \prod_{k=1}^{n(\pi)}\left\{1+x_{k}\right\} \\
= & 1+\sum_{k=1}^{n} x_{k}+\sum_{k_{2}=1}^{n} \sum_{k_{1}=1}^{k_{2}-1} x_{k_{1}} x_{k_{2}}+\cdots \\
& +\sum_{k_{r}=1}^{n} \sum_{k_{r-1}=1}^{k_{r}-1} \cdots \sum_{k_{1}=1}^{k_{2}-1} \mathrm{x}_{k_{1}} \cdots \mathrm{x}_{k_{r}}+\cdots+\mathrm{x}_{1} \mathrm{x}_{2} \cdots \mathrm{x}_{n} .
\end{aligned}
$$

Hence 


$$
\int_{a}^{b}(1+f d t)=1+\lim _{r \downarrow} \sum_{r=1}^{n(\pi)}\left\{\sum_{k_{r}=1}^{n(\pi)} \sum_{k_{r-1}-1}^{k_{r}-1} \cdots \sum_{k_{1}=1}^{k_{r}-1} x_{k_{1}} \cdots x_{k_{r}}\right\} .
$$

We must show that the limit on the R.H.S. exists. For all $r \leqq n(\pi)$ let us set

$$
U_{r}\left(\pi, \pi^{*}\right)=\sum_{k_{r}=1}^{n(\pi)} \sum_{k_{r-1}=1}^{k_{r}-1} \cdots \sum_{k_{1}=1}^{k_{2}-1} \mathrm{x}_{k_{1}} \cdots \mathrm{x}_{k_{r}}
$$

From (21.5) we then have

$$
\lim _{\pi \downarrow} U_{r}\left(\pi, \pi^{*}\right)=\int_{a}^{b} \int_{a}^{t_{r}} \cdots \int_{a}^{t_{2}} f\left(t_{1}\right) \cdots f\left(t_{r}\right) d t_{1} \cdots d t_{r}
$$

Also for all $r \leqq n(\pi)$ we have $\left|U_{r}\left(\pi, \pi^{*}\right)\right| \leqq M^{r} \sigma_{r}$, where $M=\sup _{a \leq t \leq b}|f(t)|$ and $\sigma_{r}$ is the $r$ th symmetric function of the $\left|\Delta_{k}\right|$. As is well known

$$
\sigma_{r} \leqq\left(\sum_{k=1}^{n(\pi)}\left|\Delta_{k}\right|\right)^{r} / r !=(b-a)^{r / r !} \text {. }
$$

Hence for all $r \leqq n(\pi)$

$$
\left|U_{r}\left(\pi, \pi^{*}\right)\right| \leqq M^{r}(b-a) r / r ! .
$$

Since $\sum_{r} M^{r}(b-a)^{r} / r$ ! converges, it follows from (2) and Lemma A2 (Appendix 2) that

$$
\begin{aligned}
\lim _{x \downarrow} \sum_{r=1}^{n(\pi)} U_{r}\left(\pi, \pi^{*}\right) & =\sum_{r=1}^{\infty} \lim _{x \downarrow} U_{r}\left(\pi, \pi^{*}\right) \\
& =\sum_{r=1}^{\infty} \int_{a}^{b} \int_{a}^{t_{r}} \cdots \int_{a}^{t_{2}} f\left(t_{1}\right) \cdots f\left(t_{r}\right) d t_{1} \cdots d t_{r},
\end{aligned}
$$

the series on the R.H.S. being convergent. The theorem follows from (1). (Q.E.D.)

From the duality principle we obtain the corresponding result for the $R \smile$-integral. Thus:

COROLlaRY 22.2. If $f$ is $R$-integrable on $[a, b]$ then

$$
\begin{aligned}
\int_{a}^{b}(1+f d t)=1 & +\int_{a}^{b} f d t+\int_{a}^{b} \int_{a}^{t_{2}} f\left(t_{2}\right) f\left(t_{1}\right) d t_{2} d t_{1} \\
& +\int_{a}^{b} \int_{a}^{t_{3}} \int_{a}^{t_{2}} f\left(t_{3}\right) f\left(t_{2}\right) f\left(t_{1}\right) d t_{3} d t_{2} d t_{1}+\cdots,
\end{aligned}
$$

the series on the R.H.S. being convergent.

23. Integral equation for the product integral $\left({ }^{40}\right)$. It should be noticed

$\left({ }^{10}\right)$ The method outlined below of deriving the integral equation from the Peano-series is due to Volterra and Hostinsky [1, pp. 52-53]. 
that the series

$$
\sum_{r=1}^{\infty} \int_{a}^{t} \int_{a}^{t_{r}} \cdots \int_{a}^{t_{2}} f\left(t_{1}\right) \cdots f\left(t_{r}\right) d t_{1} \cdots d t_{r}
$$

converges uniformly with respect to $t \in[a, b]$. This follows from the $M$-test since for all $t \in[a, b]$ we have

$$
\left|\int_{a}^{t} \int_{a}^{t_{r}} \cdots \int_{a}^{t_{2}} f\left(t_{1}\right) \cdots f\left(t_{r}\right) d t_{1} \cdots d t_{r}\right| \leqq M^{r}(b-a) r / r !
$$

We might add that a uniformly convergent series on $[a, b]$ remains uniformly convergent when each term is multiplied by a function bounded on $[a, b]$.

Let $Y \frown(x)=\int_{a}^{x} \frown(1+f d t)$. Applying (19.3) to the uniformly convergent series for $\boldsymbol{Y}^{\frown}(t) \cdot \boldsymbol{f}(t)$ we get

$$
\int_{a}^{x} Y \frown(t) f(t) d t=\int_{a}^{x} f(t) d t+\sum_{r=1}^{\infty} \int_{a}^{x} \int_{a}^{t_{r}} \cdots \int_{a}^{t_{2}} f\left(t_{1}\right) \cdots f\left(t_{r}\right) d t_{1} \cdots d t_{r} .
$$

Or putting $s=r+1$ and using Theorem 22.1, we get

$$
\int_{a}^{x} Y \frown(t) f(t) d t=\sum_{s=1}^{\infty} \int_{a}^{x} \int_{a}^{t_{0}} \cdots \int_{a}^{t_{2}} f\left(t_{1}\right) \cdots f\left(t_{s}\right) d t_{1} \cdots d t_{s}=Y \frown(x)-1 .
$$

We thus arrive at the integral equation $Y \frown(x)=1+\int_{a}^{x} Y \frown(t) f(t) d t$. The duality principle yields a corresponding integral equation for $Y^{\smile}(x)=\int_{a}^{x}(1+f d t)$. We thus obtain the following theorem.

THEOREM 23.1. If $\boldsymbol{f}$ is R-integrable on $[a, b]$ then the product integrals

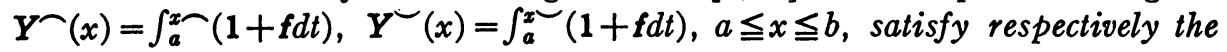
following integral equations

$$
Y \frown(x)=1+\int_{a}^{x} Y \frown(t) f(t) d t, \quad Y \smile(x)=1+\int_{a}^{x} f(t) Y \smile(t) d t .
$$

24. Commutative normed ring. The $R$-integral can be construed as a limiting product of exponential factors and the $R$-integral as a limiting sum of logarithmic terms. We accordingly have the following two lemmas, the first of which has been proved by L. Schlesinger [1, pp. 42-43]. The proof of the second we leave to the reader.

LEMma 24.1. If $f$ is $R$-integrable on $[a, b]$ then

$$
\lim _{x \downarrow} \prod_{k=1}^{n(\pi)} \operatorname{Exp}\left\{f\left(t_{k}\right)\left|\Delta_{k}\right|\right\} \text { exists and equals } \int_{a}^{b \frown}(1+f d t) .
$$

Lemma 24.2. If $\mathrm{f}$ is R-integrable on $[a, b]$ then 


$$
\left.\lim _{x \downarrow} \sum_{k=1}^{n(\pi)} \operatorname{Ln}\left\{1+f\left(t_{k}\right)\left|\Delta_{k}\right|\right)\right\} \text { exists and equals } \int_{a}^{b} f d t .
$$

These lemmas yield nice results for integration in a commutative normed ring $X$. In view of (3.8) and (3.5), Lemma 24.1 yields for such a ring

$$
\int_{a}^{b}(1+f d t)=\lim _{\pi \downarrow} \operatorname{Exp}\left\{\sum_{k=1}^{n(\pi)} f\left(t_{k}\right)\left|\Delta_{k}\right|\right\}=\operatorname{Exp}\left\{\int_{a}^{b} f d t\right\} .
$$

Likewise from Lemma 24.2 we get

$$
\int_{a}^{b} f d t=\operatorname{Ln} \int_{a}^{b}(1+f d t)
$$

This proves the following theorem.

THEOREM 24.3. If $X$ is commutative then for every $R$-integrable function $\boldsymbol{f}$ we have

$$
\int_{a}^{b}(1+f d t)=\operatorname{Exp}\left\{\int_{a}^{b} f d t\right\}, \quad \int_{a}^{b} f d t=\operatorname{Ln}\left\{\int_{a}^{b}(1+f d t)\right\} .
$$

Thus in the commutative case the Peano series reduces to the exponential series.

\section{Differentiation}

We shall now define additive and multiplicative differentiation of functions on a real domain with values in a normed ring $X$. After indicating the elementary properties of the derivative we shall turn to the so-called fundamental problem of the integral calculus. We shall conclude with a discussion of integration by parts and changing the variable.

\section{The derivatives defined.}

Definition 25.1. If $\boldsymbol{f}$ is a function on $[a, b]$ into $X$ then we shall say that $f$ is $R$-derivable at $x \in[a, b]$ if and only if

$$
\lim _{\Delta_{x \rightarrow 0, x+\Delta_{x} \in[a, b]}} \frac{1}{\Delta_{x}}\left\{f\left(x+\Delta_{x}\right)-f(x)\right\}
$$

exists. The limit will be called the $R$-derivative of $f$ at $x$ and denoted by $f^{\circ}(x)$.

Definition 25.2. If $\boldsymbol{f}$ is a function on $[a, b]$ into $X$ then we shall say that $f$ is $R$-derivable at $x \in[a, b]$ if and only if ${ }^{(41)}$

$$
\lim _{\Delta_{x} \rightarrow 0, x+\Delta_{x} \in[a, b]} \frac{1}{\Delta_{x}}\left\{f^{-1}(x) f\left(x+\Delta_{x}\right)-1\right\}
$$

(11) Here and throughout the sequel $f^{-1}(x)$ will abbreviate the inverse $\{f(x)\}^{-1}$, whenever this exists. 
exists. The limit will be called the $R \frown$-derivative of $f$ at $x$ and denoted by

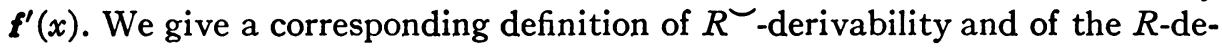
rivative $f^{\prime}$.

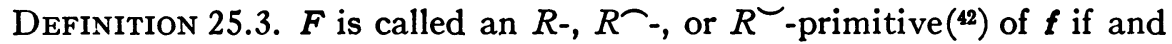

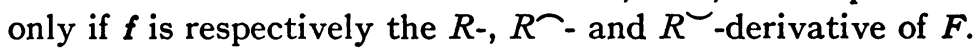

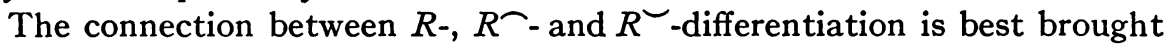
out by the following lemma which obviously follows from the definition.

LemMa 25.4. $f$ is $R$-derivable or $R \smile$-derivable at $x$ if and only if $f$ is $R$-derivable at $x$ and $f(x)$ has an inverse. In this case we can state the connection between $R-, R^{\frown}$ - and $R^{\smile}$-derivative in the following equivalent ways:

$$
\begin{array}{lll}
f^{\prime}(x)=f^{-1}(x) f^{\bullet}(x), & f^{\prime}(x)=f^{-1}(x) f^{\prime}(x) f(x), \\
f^{\prime}(x)=f^{\cdot}(x) f^{-1}(x), & f^{\prime}(x)=f(x) f^{\prime}(x) f^{-1}(x) .
\end{array}
$$

26. Duality. Since multiplication in $X$ is in general noncommutative it follows that in general $f^{\prime}(x) \neq f^{\prime}(x)$. The duality principle $(\$ 7)$ however enables us to infer a result concerning $f^{\prime}$ from the corresponding result concerning $f^{\prime}$. We shall therefore state and prove results for $R$-derivatives only.

27. Elementary properties of the derivatives. As in the real case we can easily establish the following properties for $R$-differentiation.

LeMma 27.1. (i) $f=0$ on $[a, b]$ if and only if $f$ is constant on $[a, b]$.

(ii) $(c f)^{\circ}=c f^{\circ}, c \in X$ or $c$ a complex number.

(iii) $(f+g)^{\circ}=f^{\circ}+g^{\circ}$.

(iv) $\left(f \xi^{\circ}\right)^{\circ}=f g^{\circ}+f^{\circ} \xi^{\circ}$

$$
\begin{aligned}
\left(f^{-1}\right)^{\cdot} & =-f^{-1} f^{\cdot} f^{-1} \\
& =f^{-2} \cdot f \text { in a commutative ring } X .
\end{aligned}
$$

From this and Lemma 25.4 we easily infer the corresponding properties for $R$ - -differentiation (cf. Schlesinger [1, pp. 47-49]). Thus:

Lemma 27.2. It will be assumed that $\mathrm{f}^{-1}(x), \boldsymbol{g}^{-1}(x), c^{-1}$ exist.

(i) $\boldsymbol{f}=$ constant on $[a, b]$ and $\boldsymbol{f}^{-1}$ exists if and only if $\boldsymbol{f}^{\prime}=0$.

(ii) $(\mathrm{cf})^{\prime}=\boldsymbol{f}^{\prime},(\mathrm{fc})^{\prime}=\mathrm{c}^{-1} \boldsymbol{f}^{\prime} \mathrm{c}, \mathrm{c} \in X$ or $c$ a complex number.

(iii) $(f+g)^{\prime}=(f+g)^{-1}\left\{f f^{\prime}+g g^{\prime}\right\}$.

$$
\begin{aligned}
(f \boldsymbol{g})^{\prime} & =\boldsymbol{g}^{-1} \boldsymbol{f}^{\prime} \boldsymbol{g}+\boldsymbol{g}^{\prime} \\
& =\boldsymbol{f}^{\prime}+\boldsymbol{g}^{\prime} \text { in a commutative ring } X .
\end{aligned}
$$

(v) $\left(f^{-1}\right)^{\prime}=-f^{\prime}$.

(vi) If $\boldsymbol{f}^{\prime}=\boldsymbol{g}^{\prime}$ on $[a, b]$ then $\boldsymbol{f}=\mathbf{c} \cdot \boldsymbol{g}$, where $c \in X$.

28. Reciprocity of integration and differentiation. With regard to the question of the reciprocity of differentiation and integration our theory is no

(43) The term "anti-derivative" is more common. 
weaker than the classical Riemann theory; in other words we can show that the integral differentiates to the integrand when this is continuous, and that for every integrable function possessing a primitive, the integral is the primitive up to an arbitrary constant.

Let $F(x)=\int_{a}^{x} f d t, a \leqq x \leqq b$. As in the real case we have

$$
\begin{aligned}
\left|\frac{1}{\Delta_{x}}\left\{F\left(x_{0}+\Delta_{x}\right)-F\left(x_{0}\right)\right\}-f\left(x_{0}\right)\right| & =\left|\frac{1}{\Delta_{x}} \int_{x_{0}}^{x_{0}+\Delta_{x}}\left\{f(t)-f\left(x_{0}\right)\right\} d t\right| \\
& \leqq \sup _{t \in\left[x_{0}, x_{0}+\Delta_{x}\right]}\left|f(t)-f\left(x_{0}\right)\right|
\end{aligned}
$$

from (12.6) and (18.1). If now $f$ is continuous at $x_{0}$ then the R.H.S. can be made arbitrarily small by making $\left|\Delta_{x}\right|$ small enough. Hence $F^{\prime}\left(x_{0}\right)=f\left(x_{0}\right)$. Thus:

THEOREM 28.1. If $F(x)=\int_{a}^{x} f d t, a \leqq x \leqq b$, then $f$ is continuous at $x_{0}$ implies $F$ is $R$-derivable at $x_{0}$ and $F^{*}\left(x_{0}\right)=f\left(x_{0}\right)$.

Let next $F(x)=\int_{a}^{x} \frown(1+f d t)$. We know (cf. Theorem 23.1) that $F$ satisfies the integral equation $F(x)=1+\int_{a}^{x} F(t) f(t) d t$. If $f$ is continuous at $x_{0}$ then by the last theorem

$$
F \cdot\left(x_{0}\right)=\left\{\int_{a}^{x} F(t) f(t) d t\right\}_{x=x_{0}}^{\cdot}=F\left(x_{0}\right) f\left(x_{0}\right) .
$$

Also we know (cf. Theorem 14.1) that $F\left(x_{0}\right)$ has an inverse. Hence

$$
F^{\prime}\left(x_{0}\right)=F^{-1}\left(x_{0}\right) F^{\cdot}\left(x_{0}\right)=f\left(x_{0}\right) .
$$

We thus obtain the following theorem.

TheOREM 28.2. If $F(x)=\int_{a}^{x}-(1+f d t), a \leqq x \leqq b$, then $f$ is continuous at $x_{0}$ implies that $F$ is $R$-differentiable at $x_{0}$ and $F^{\prime}\left(x_{0}\right)=f\left(x_{0}\right)$.

The following theorem, the best of its kind available for $R$-integration, is due to T. H. Hildebrandt (see L. Graves [1, pp. 171-172]).

THEOREM 28.3. If $F$ is an $R$-primitive of $f$, and $f$ is $R$-integrable on $[a, b] '$ then for all $x \in[a, b], F(x)=F(a)+\int_{a}^{x} f d t$.

We shall apply Hildebrandt's method to prove the corresponding theorem for $R$-integration. The following lemmas are needed. We leave their proofs to the reader.

Lemma 28.4. If $f$ is $R$-derivable at $x$ then

$$
\lim _{h, h^{\prime} \rightarrow 0+0} \frac{1}{h+h^{\prime}}\left\{f(x+h)-f\left(x-h^{\prime}\right)\right\}=f^{\cdot}(x) .
$$


Lemma 28.5. If $f$ is $R$-derivable at $x$ then

$$
\lim _{h, h^{\prime} \rightarrow 0+0} \frac{1}{h+h^{\prime}}\left\{f^{-1}\left(x-h^{\prime}\right) f(x+h)-1\right\}=f^{\prime}(x) .
$$

THEOREM 28.6. If $F$ is an $R$-primitive of $f$ and $f$ is $R$-integrable on $[a, b]$ then for all $x \in[a, b], F(x)=F(a) \int_{a}^{x}(1+f d t)$.

Proof. We shall prove the result for $x=b$. The same method will work for $x<b$. Let $\epsilon>0$. Since $\boldsymbol{f}$ is $R$-integrable there exists $\delta>0$ such that for all $\pi$ with $|\pi|<\delta$ and all valuations $\pi^{*}$ we have

$$
\left|J \frown\left(f, \pi, \pi^{*}\right)-\int_{a}^{b \frown}(1+f d t)\right| \leqq \epsilon / 2 .
$$

Since $f$ is the $R$-derivative of $F$ on $[a, b]$ we know from the last lemma that for all $z \in[a, b]$ there exists a closed subinterval $\sigma_{z}$ such that $\left|\sigma_{z}\right|<\delta$, $z$ is the middle point of $\sigma_{z}$ unless $z=a$ or $z=b$ in which case it is the left or right end point of $\sigma_{z}$, and such that for all $x, y \in \sigma_{z}, x \leqq z \leqq y$ we have

$$
\left|\frac{1}{y-x}\left\{F^{-1}(x) F(y)-1\right\}-f(z)\right| \leqq \frac{\exp (-M(b-a))}{2 L(b-a)\left|F^{-1}(a)\right|} \epsilon,
$$

where $M=\sup _{a \leqq t \leqq b}|f(t)|$ and $L=\sup _{a \leqq t \leqq b}|F(t)| 0$.

The family $\mathcal{N}$ of neighbourhoods $\sigma_{z}$ is a closed covering of $[a, b]$. By the Heine-Borel theorem there exists $\mathcal{N}_{0}=\left\{\sigma_{z_{1}}, \cdots, \sigma_{z_{n}}\right\} \subset \mathcal{N}$ which also covers $[a, b]$. We may assume that no subinterval $\sigma_{z_{k}}$ is wholly contained in another such subinterval, and that $z_{1}=a$ and $\sigma_{z_{n}}$ ends at $b$. Applying (11.1) we get a partition $\pi_{0}=\left\{\Delta_{1}, \cdots, \Delta_{n}\right\} \in \Pi_{a}^{b}$ such that $z_{k} \in \Delta_{k} \subset \sigma_{z_{k}}$. In other words we have a partition $\pi_{0}$ of $[a, b]$ such that $\left|\pi_{0}\right|<\delta$ and if $\Delta_{k}=\left[x_{k-1}, x_{k}\right]$ then

$$
\left|F\left(x_{k-1}\right) f\left(z_{k}\right)\right| \Delta_{k}\left|-\left\{F\left(x_{k}\right)-F\left(x_{k-1}\right)\right\}\right| \leqq \frac{\exp (-M(b-a))}{2(b-a)\left|F^{-1}(a)\right|} \epsilon\left|\Delta_{k}\right| .
$$

Let now $\pi_{0}^{*}$ be the valuation of $\pi_{0}$ consisting of $z_{k}$ from $\Delta_{k}$. Then from (4.5) we get

$$
\begin{aligned}
& \left|J \sim\left(f, \pi, \pi^{*}\right)-F^{-1}(a) F(b)\right| \\
& \quad \leqq \exp \left(\sum_{1}^{n}\left|f\left(z_{k}\right)\right|\left|\Delta_{k}\right|\right) \sum_{k=1}^{n}\left\{\left|F^{-1}(a) F\left(x_{k-1}\right) f\left(z_{k}\right)\right| \Delta_{k} \mid\right. \\
& \left.\quad-F^{-1}(a)\left\{F\left(x_{k}\right)-F\left(x_{k-1}\right)\right\} \mid \exp \left(-\sum_{i=1}^{k}\left|f\left(z_{i}\right)\right|\left|\Delta_{i}\right|\right)\right\} \\
& \quad \leqq \exp (M(b-a))\left|F^{-1}(a)\right| \sum_{k=1}^{n}\left|F\left(x_{k-1}\right) f\left(z_{k}\right)\right| \Delta_{k}\left|-\left\{F\left(x_{k}\right)-F\left(x_{k-1}\right)\right\}\right| \\
& \quad \leqq \epsilon / 2
\end{aligned}
$$


from (2). In view of (1) and (3), the triangle inequality yields

$$
\left|\int_{a}^{b \sim}(1+f d t)-F^{-1}(a) F(b)\right|<\epsilon .
$$

And since $\epsilon$ is arbitrary, the theorem follows. (Q.E.D.)

29. Integration by parts. As in the real case the following familiar rule of integration by parts holds.

(29.1) If $f$ and $\phi$ are $R$-derivable on $[a, b]$ and $f$ and $\phi^{\bullet}$ are $R$-integrable on $[a, b]$ then

$$
\int_{a}^{b} f \cdot \phi d t+\int_{a}^{b} f \phi \cdot d t=f(b) \phi(b)-f(a) \phi(a) .
$$

The following theorem is a simple generalization of this.

THEOREM 29.2. If $f$ and $\phi$ are $R$-integrable on $[a, b]$ then

$$
\int_{a}^{b}\left\{\int_{a}^{t} f d x\right\} \phi(t) d t+\int_{a}^{b} f(t)\left\{\int_{a}^{t} \phi d x\right\} d t=\int_{a}^{b} f d t \cdot \int_{a}^{b} \phi d t .
$$

Proof. In view of the hypothesis and Theorem 21.3 all the integrals in question exist. The result is now a consequence of (21.5) and the identity

$$
\sum_{k=1}^{n}\left(\sum_{i=1}^{k} x_{i}\right) y_{k}+\sum_{k=1}^{n} x_{k}\left(\sum_{i=1}^{k-1} y_{i}\right)=\sum_{k=1}^{n} x_{k} \cdot \sum_{k=1}^{n} y_{k} \text {. }
$$

(Q.E.D.)

Turning to the corresponding theory for $R$-integration which is naturally harder, we obtain the following result the proof of which we shall leave to the reader.

(29.3) If $f$ and $\phi$ are $R$-derivable on $[a, b]$ and $f^{\prime}, \phi^{\prime}$ are $R$-integrable on $[a, b]$ then

$$
\int_{a}^{b \sim}\left\{1+\left(f^{\prime}+\phi^{\prime}\right) d t\right\}=\phi^{-1}(a) \cdot \int_{a}^{b}\left\{1+\phi^{\prime} \phi^{-1} d t\right\} \cdot \phi(a) \cdot \int_{a}^{b \frown}\left(1+\phi^{\prime} d t\right) .
$$

This suggests the following generalization corresponding to Theorem 29.2:

$$
\begin{aligned}
\int_{a}^{b} & \{1+(f+\phi) d t\} \\
= & \int_{a}^{b}\left[1+\left\{\int_{a}^{b}(1+\phi d t)\right\} f(t)\left\{\int_{a}^{b}(1+\phi d t)\right\}^{-1} d t\right] \cdot \int_{a}^{b}(1+\phi d t),
\end{aligned}
$$

where $f$ and $\phi$ are any $R$-integrable functions $\left({ }^{43}\right)$. To establish this we need two lemmas.

(43) The existence of $\left\{\int_{0}^{t} \sim(1+\phi d t)\right\}^{-1}$ is guaranteed by Theorem 14.1. 
LemMa 29.4(44). If $\phi$ is $R$-integrable on $[a, b]$ and $f$ is bounded then

$$
\begin{aligned}
\lim _{x \downarrow} \mid \prod_{k=1}^{n} & {\left[1+\left\{\prod_{i=1}^{k}\left(1+\phi\left(t_{i}\right)\left|\Delta_{i}\right|\right)\right\} f\left(t_{k}\right)\left\{\prod_{i=1}^{k}\left(1+\phi\left(t_{i}\right)\left|\Delta_{i}\right|\right)\right\}^{-1}\left|\Delta_{k}\right|\right] } \\
& -\prod_{k=1}^{n}\left[1+\left\{\int_{a}^{t_{k}}(1+\phi d x)\right\} f\left(t_{k}\right)\left\{\int_{a}^{t_{k}}(1+\phi d x)\right\}^{-1}\left|\Delta_{k}\right|\right] \mid=0 .
\end{aligned}
$$

Proof. Let $K, L, M$ denote positive constants independent of $\pi$ and $\pi^{*}$. Applying 4.43 to the absolute difference on the L.H.S. which we shall denote by $D\left(\pi, \pi^{*}\right)$ we get in view of Corollary 11.7 and (3.4)

$$
\begin{aligned}
D\left(\pi, \pi^{*}\right) \leqq & \exp k \cdot \sum_{k=1}^{n} \mid \prod_{i=1}^{k}\left(1+\phi\left(t_{i}\right)\left|\Delta_{i}\right|\right) \cdot f\left(t_{k}\right)\left\{\prod_{i=1}^{k}\left(1+\phi\left(t_{i}\right)\left|\Delta_{i}\right|\right)\right\}^{-1} \\
& -\int_{a}^{t_{k}}(1+\phi d x) \cdot f\left(t_{k}\right)\left\{\int_{a}^{t_{k}}(1+\phi d x)\right\}^{-1}|| \Delta_{k} \mid
\end{aligned}
$$

or, say,

$$
D\left(\pi, \pi^{*}\right) \leqq \exp k \cdot \sum_{k=1}^{n(\pi)} a_{\pi, \pi^{*}}^{k}\left|\Delta_{k}\right| .
$$

For all $\pi$ and valuations $\pi^{*}$ let us define $g\left(\pi, \pi^{*}, x\right)$ as the positive stepfunction on $[a, b]$ such that for all $x \in \Delta_{k} \in \pi, g\left(\pi, \pi^{*}, x\right)=a_{\pi, \pi^{*}}^{\boldsymbol{k}}$. Clearly $g\left(\pi, \pi^{*}, x\right)<L$. Also for a given $x \in[a, b]$ and $\pi \in \Pi_{a}^{b}$, letting $\Delta_{k(x)}=\left[x_{k(\pi)-1}\right.$, $\left.x_{k(x)}\right]$ be the subinterval of $\pi$ containing $x$, we have

$$
\begin{aligned}
g\left(\pi, \pi^{*}, x\right) \leqq & \left|\prod_{i=1}^{k(x)}\left(1+\phi\left(t_{i}\right)\left|\Delta_{i}\right|\right)-\int_{a}^{t_{k}(x) \frown}(1+\phi d x)\right| \cdot\left|f\left(t_{k(x)}\right)\right| \\
& +\left|\left\{\prod_{i=1}^{k(\pi)}\left(1+\phi\left(t_{i}\right)\left|\Delta_{i}\right|\right)\right\}^{-1}\right| \\
& -\left\{\int_{a}^{t_{k}(x) \frown}(1+\phi d x)|\cdot| f\left(t_{k(x)}\right)|\cdot|\left\{\prod_{i=1}^{k(\pi)}\left(1+\phi\left(t_{i}\right)\left|\Delta_{i}\right|\right)\right\}^{-1}\right. \\
\leqq & M\left[\left|\prod_{i=1}^{k(\pi)}\left(1+\phi\left(t_{i}\right)\left|\Delta_{i}\right|\right)-\int_{a}^{t_{k}(\pi)-}(1+\phi d x)\right|\right. \\
+ & \left.\left|\left\{\prod_{i=1}^{k(x)}\left(1+\phi\left(t_{i}\right)\left|\Delta_{i}\right|\right)\right\}^{-1}-\left\{\int_{a}^{t_{k}(x) \frown}(1+\phi d x)\right\}^{-1}\right|\right] .
\end{aligned}
$$

Since as $\pi \downarrow, x_{k(\pi)-1}, x_{k(\pi)}$ and therefore $t_{k(\pi)}$, approach $x$, and $\phi$ is $R$-integrable it follows from (18.2) and (3.4) that $\lim _{\pi \downarrow} g\left(\pi, \pi^{*}, x\right)=0$. Applying Lemma

(4) This and (21.2) are of a similar nature. Their proofs are based on similar ideas. 
A1.2 (Appendix 1) we get $\lim _{\pi \downarrow} \sum_{k=1}^{n(x)} a_{\pi, \pi^{*}}^{k}\left|\Delta_{k}\right|=0$. The lemma now follows from (1). (Q.E.D.)

LEMMA 29.5

$$
\begin{aligned}
\prod_{k=1}^{n}\left[1+\left\{\prod_{i=1}^{k}\left(1+y_{i}\right)\right\} x_{k}\left\{\prod_{i=1}^{k}\left(1+y_{i}\right)\right\}^{-1}\right] & \cdot \prod_{k=1}^{n}\left(1+y_{k}\right) \\
& =\prod_{k=1}^{n}\left\{\left(1+y_{k}\right)\left(1+x_{k}\right)\right\} .
\end{aligned}
$$

Proof. We shall use induction. The result certainly holds for $n=1$. Suppose it holds for $n$. Denoting the R.H.S. by $C(n)$ and the two products on the L.H.S. by $A(n)$ and $B(n)$ we have

$$
\begin{aligned}
A(n+1) \cdot & B(n+1) \\
= & C(n) B^{-1}(n)\left[1+\left\{\prod_{i=1}^{n+1}\left(1+y_{i}\right)\right\} x_{n+1}\left\{\prod_{i=1}^{n+1}\left(1+y_{i}\right)\right\}^{-1}\right] \\
& \cdot B(n) \cdot\left(1+y_{n+1}\right) \\
= & C(n)\left[1+\left(1+y_{n+1}\right) x_{n+1}\left(1+y_{n+1}\right)^{-1}\right]\left(1+y_{n+1}\right) \\
= & C(n)\left[\left(1+y_{n+1}\right)\left(1+x_{n+1}\right)\right] \\
= & C(n+1) .
\end{aligned}
$$

The result follows by induction. (Q.E.D.)

THEOREM 29.6. If $f$ and $\phi$ are $R$-integrable on $[a, b]$ then so is

and

$$
\int_{a}^{\mathfrak{r}}(1+\phi d x) \cdot f(t) \cdot\left\{\int_{a}^{n}(1+\phi d x)\right\}^{-1} \quad(a \leqq t \leqq b)
$$

$$
\begin{aligned}
\int_{a}^{b \sim}\left[1+\left\{\int_{a}^{t}(1+\phi d x)\right\} f(t)\left\{\int_{a}^{t}(1+\phi d x)\right\}^{-1} d t\right] \cdot \int_{a}^{b \sim}(1+\phi d t) \\
=\int_{a}^{b \frown}\{1+(f+\phi) d t\} .
\end{aligned}
$$

Proof. From Lemma 29.4 we know that given $\epsilon>0$ there exists $\pi_{0} \in \Pi_{a}^{b}$ such that for all $\pi<\pi_{0}$ and all valuations $\pi^{*}$ we have

$$
\begin{aligned}
\mid \prod_{k=1}^{n(x)}[1+ & \left.\left\{\prod_{i=1}^{k}\left(1+\phi\left(t_{i}\right)\left|\Delta_{i}\right|\right)\right\} f\left(t_{k}\right)\left\{\prod_{i=1}^{k}\left(1+\phi\left(t_{i}\right)\left|\Delta_{i}\right|\right)\right\}^{-1}\left|\Delta_{k}\right|\right] \\
& -J^{-}\left(\int_{a}^{\mathfrak{L}}(1+\phi d x) \cdot f(t) \cdot\left\{\int_{a}^{\curvearrowleft}(1+\phi d x)\right\}^{-1}, \pi, \pi^{*}\right) \mid<\epsilon / 2 .
\end{aligned}
$$

Hence 


$$
\begin{aligned}
& \mid J \frown\left(\int_{a}^{\mathfrak{L}}(1+\phi d x) \cdot f(t)\left\{\int_{a}^{\curvearrowleft}(1+\phi d x)\right\}^{-1}, \pi, \pi^{*}\right) \cdot J \frown\left(\phi, \pi, \pi^{*}\right) \\
& -J \sim\left(f+\phi, \pi, \pi^{*}\right) \\
& \leqq \mid \prod_{k=1}^{n}\left[1+\prod_{i=1}^{k}\left(1+\phi\left(t_{i}\right)\left|\Delta_{i}\right|\right) f\left(t_{k}\right)\left\{\prod_{i=1}^{k}\left(1+\phi\left(t_{i}\right)\left|\Delta_{i}\right|\right)\right\}^{-1}\left|\Delta_{k}\right|\right] \\
& \cdot \prod_{k=1}^{n}\left(1+\phi\left(t_{k}\right)\left|\Delta_{k}\right|\right)-J \frown\left(f+\phi, \pi, \pi^{*}\right) \mid+\epsilon / 2 \\
& \leqq\left|\prod_{k=1}^{n}\left[\left\{1+\phi\left(t_{k}\right)\left|\Delta_{k}\right|\right\}\left\{1+f\left(t_{k}\right)\left|\Delta_{k}\right|\right\}\right]-J\left(f+\phi, \pi, \pi^{*}\right)\right|+\epsilon / 2 \\
& \leqq \exp \left(\left\{M_{f}+M_{\phi}\right\}(b-a)\right) \cdot\left[\exp \left(M_{\phi}+M_{f}(b-a)|\pi|\right)-1\right]+\epsilon / 2
\end{aligned}
$$

from Lemma 29.5 and (4.45), where $M_{f}=\sup _{a \leqq t \leqq b}|f(t)|$ and $M_{\phi}=\sup _{a \leqq t \leq b}$ $|\phi(t)|$. Since the first term on the R.H.S. of the last inequality can be made less than $\epsilon / 2$ for all $\pi \prec \pi_{1}$ say, it follows that

$$
\begin{aligned}
\lim _{\pi \downarrow} \mid J^{-}\left(\int_{a}^{\curvearrowleft}(1+\phi d x) \cdot f(t) \cdot\left\{\int_{a}^{\curvearrowleft}(1+\phi d x)\right\}^{-1}, \pi, \pi^{*}\right) \cdot J \frown\left(\phi, \pi, \pi^{*}\right) \\
-J \frown\left(f+\phi, \pi, \pi^{*}\right) \mid=0 .
\end{aligned}
$$

Since $\phi$ and $f+\phi$ are $R$-integrable, it follows from this equation that so is

and

$$
\int_{a}^{\mathfrak{t}}(1+\phi d x) \cdot f(t) \cdot\left\{\int_{a}^{t}(1+\phi d x)\right\}^{-1}
$$

$$
\begin{aligned}
\int_{a}^{b}\left[1+\left\{\int_{a}^{t}(1+\phi d x)\right\} f(t)\left\{\int_{a}^{t}(1+\phi d x)\right\}^{-1} d t\right] \cdot \int_{a}^{b}(1+\phi d t) \\
=\int_{a}^{b \frown}[1+(f+\phi) d t] .
\end{aligned}
$$

(Q.E.D.)

For a commutative ring this result reduces to

$$
\int_{a}^{b \frown}(1+f d t) \cdot \int_{a}^{b}(1+\phi d t)=\int_{a}^{b \sim}[1+(f+\phi) d t],
$$

which is also obvious since in the commutative case (cf. Theorem 24.3)

$$
\int_{a}^{b \frown}(1+f d t)=\operatorname{Exp}\left(\int_{a}^{b} f d t\right)
$$

It should be noticed that even in the non-commutative case we have 


$$
\begin{aligned}
\int_{a}^{b \frown}\left[1+\left\{\int_{a}^{t}(1+\phi d x)\right\} f(t)\left\{\int_{a}^{t}(1+\phi d x)\right\}^{-1} d t\right] \cdot \int_{a}^{b}(1+\phi d x) \\
=\int_{a}^{b \frown}\left[1+\left\{\int_{a}^{t \frown}(1+f d x)\right\} \phi(t)\left\{\int_{a}^{t \frown}(1+f d x)\right\}^{-1} d t\right] \\
\cdot \int_{a}^{b}(1+f d t) .
\end{aligned}
$$

30. Change of variable. We shall now extend the familiar and useful rule of substitution of ordinary integration.

THEOREM 30.1. If $f$ is $R$-integrable on $[a, b]$ and $\lambda$ is a strictly increasing real function on $[\alpha, \beta]$ with $\lambda(\alpha)=a, \lambda(\beta)=b\left({ }^{45}\right)$ and with a derivative $\lambda^{\cdot}, R$-integrable on $[\alpha, \beta]$, then the function $f\{\lambda(x)\} \cdot \lambda^{\circ}(x)$ is R-integrable on $[\alpha, \beta]$ and

$$
\int_{a}^{b \sim}\{1+f(s) d s\}=\int_{\alpha}^{\beta \sim}\left[1+f\{\lambda(t)\} \cdot \lambda^{\cdot}(t) d t\right] .
$$

Proof. Let $\pi_{\alpha}^{\beta}=\left\{\Delta_{1}, \cdots, \Delta_{n}\right\} \in \Pi_{\alpha}^{\beta}$ where $\Delta_{k}=\left[x_{k-1}, x_{k}\right]$. The function $\lambda$ maps $\pi_{\alpha}^{\beta}$ into a partition $\pi_{a}^{b}=\left\{\nabla_{1}, \cdots, \nabla_{n}\right\} \in \Pi_{a}^{b}$ such that $\nabla_{k}=\left[\lambda\left(x_{k-1}\right), \lambda\left(x_{k}\right)\right]$. Also every valuation $\left(\pi_{\alpha}^{\beta}\right)^{*}=\left\{t_{1}, \cdots, t_{n}\right\}$ of $\pi_{\alpha}^{\beta}$ is carried into a valuation $\left(\pi_{a}^{b}\right)^{*}=\left\{s_{1}, \cdots, s_{n}\right\}$ of $\pi_{a}^{b}$ such that $s_{k}=\lambda\left(t_{k}\right)$. Hence

$$
\begin{aligned}
J \mathcal{( f , \pi _ { a } ^ { b } , \pi _ { a } ^ { b _ { * } } )} & =\prod_{k=1}^{n}\left\{1+f\left(s_{k}\right)\left|\nabla_{k}\right|\right\} \\
& =\prod_{k=1}^{n}\left\{1+f\left(s_{k}\right) \cdot\left|\lambda\left(x_{k}\right)-\lambda\left(x_{k-1}\right)\right|\right\} \\
& =\prod_{k=1}^{n}\left[1+f\left\{\lambda\left(t_{k}\right)\right\} \cdot \lambda^{\cdot}\left(\tau_{k}\right)\left|\Delta_{k}\right|\right]
\end{aligned}
$$

by the mean-value theorem, $\tau_{k}$ being some appropriate point in $\Delta_{k}$. Hence

(1)

$$
\begin{aligned}
\left|J \frown\left(f\{\lambda(t)\} \lambda \cdot(t), \pi_{\alpha}^{\beta}, \pi_{\alpha}^{\beta *}\right)-J \mathcal{f}\left(f(s), \pi_{a}^{b}, \pi_{a}^{b *}\right)\right| \\
\quad=\left|\prod_{k=1}^{n}\left[1+f\left\{\lambda\left(t_{k}\right)\right\} \lambda \cdot\left(t_{k}\right)\left|\Delta_{k}\right|\right]-\prod_{k=1}^{n}\left[1+f\left\{\lambda\left(t_{k}\right)\right\} \lambda^{\cdot}\left(\tau_{k}\right)\left|\Delta_{k}\right|\right]\right| \\
\quad \leqq \exp \left(2 M_{1} M_{2}(b-a)\right) M_{1} \sum_{k=1}^{n} \operatorname{Osc}\left(\lambda^{*}, \Delta_{k}\right)\left|\Delta_{k}\right|
\end{aligned}
$$

from (4.43), where $M_{1}=\sup _{a \leq s \leq b}|f(s)|$ and $M_{2}=\sup _{\alpha \leq t \leq \beta}\left|\lambda^{\cdot}(t)\right|$.

Now $\lambda^{\cdot}$ is $R$-integrable on $[\alpha, \beta]$ hence there is $\pi_{\alpha}^{\beta}$ such that $\left|S\left(\lambda^{*}, \pi_{\alpha}^{\beta}\right)\right|<\epsilon$. But since $\lambda^{\circ}$ is real, $\left|S\left(\lambda^{\bullet}, \pi_{\alpha}^{\beta}\right)\right|=\sum_{1}^{n}$ Osc $\left(\lambda^{\circ}, \Delta_{k}\right)\left|\Delta_{k}\right|$. It follows from (1)

(45) That is, the range of $\lambda$ is precisely $[a, b]$. 
and (9.6) that

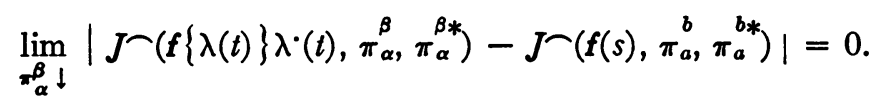

The theorem follows in view of the $R$-integrability of $f$. (Q.E.D.)

By a similar method it is easy to obtain the corresponding result for $R$-integration. Thus:

THEOREM 30.2. Under the hypothesis of Theorem 30.1 we have

$$
\int_{a}^{b} f(s) d s=\int_{\alpha}^{\beta} f\{\lambda(t)\} \lambda^{\cdot}(t) d t .
$$

We leave the proof to the reader.

\section{APPENDIX I. A LEMMA CONCERNING REAL INTEGRATION}

We need a lemma to enable us to handle limits like $\lim _{\pi \downarrow} \sum_{k-1}^{n(\pi)} a_{\pi, \pi^{*}}^{k}\left|\Delta_{k}\right|$, where $a_{\pi, \pi^{*}}^{k}$ is a positive real constant depending on $\pi$, its valuation $\pi^{*}$, and the position index $k(k=1, \cdots, n(\pi))$. The crucial point in the proof of the lemma is the use of Lebesgue's theorem on the bounded convergence of measurable functions (see Hobson [1, p. 581]).

LEMma A1.1. If (i) $\left(\pi_{n}\right)_{n=1}^{\infty} \subset \Pi_{a}^{b}$ and $\lim _{n \rightarrow \infty}\left|\pi_{n}\right|=0$;

(ii) For all $\pi_{n}$ and all valuations $\pi_{n}^{*}, g\left(\pi_{n}, \pi_{n}^{*}, x\right)$ is a positive step-function on $[a, b]$ such that for all $x \in \Delta_{k} \in \pi_{n}, g\left(\pi_{n}, \pi_{n}^{*}, x\right)=a_{\pi_{n}, x_{n}^{*}}^{k}, a$ positive constant;

(iii) $g\left(\pi_{n}, \pi_{n}^{*}, x\right)$ is uniformly bounded for all $x \in[a, b]$ and all $n$, that is, $g\left(\pi_{n}, \pi_{n}^{*}, x\right)<K$;

(iv) $\lim _{n \rightarrow \infty} g\left(\pi_{n}, \pi_{n}^{*}, x\right)=0$ for all $x \in[a, b]\left({ }^{46}\right)$; then $\left({ }^{47}\right)$

$$
\lim _{n \rightarrow \infty} \sum_{k=1}^{p(n)} a_{x_{n}, \pi_{n}^{*}}^{k}\left|\Delta_{k}\right|=0
$$

Proof. Each $g\left(\pi_{n}, \pi_{n}^{*}, x\right)$ is $L$-integrable on $[a, b]$ since it is a bounded stepfunction. Let $G\left(\pi_{n}, x\right)=\sup _{\pi_{n}^{*}} g\left(\pi_{n}, \pi_{n}^{*}, x\right)$. Then $G\left(\pi_{n}, x\right)$ is itself a stepfunction on $[a, b]$ with steps on the subintervals of $\pi_{n}$. Also from (iii)

$$
0 \leqq G\left(\pi_{n}, x\right) \leqq K .
$$

Hence for all $n, G\left(\pi_{n}, x\right)$ is $L$-integrable. Finally

$$
G\left(\pi_{n}, x\right)=\left\{G\left(\pi_{n}, x\right)-g\left(\pi_{n}, \pi_{n}^{*}, x\right)\right\}+g\left(\pi_{n}, \pi_{n}^{*}, x\right) .
$$

In view of (iv), however, we know that for each $x \in[a, b]$ and every $\epsilon>0$ there exists $n_{\epsilon, x}$ such that $n \geqq n_{\epsilon, x}$ implies $g\left(\pi_{n}, \pi_{n}^{*}, x\right)<\epsilon$. Hence for

${ }^{\left({ }^{46}\right)}$ Recourse to the Lebesgue theory may be avoided if we make the stronger assumption that $\lim _{n \rightarrow \infty} g\left(\pi_{n}, \pi_{n}^{*}, x\right)=0$, uniformly for $x \in[a, b]$. In applications, however, the demonstration of uniformity proves inconvenient.

(47) $p(n)$ denotes the number of subintervals in $\pi_{n}$. 
each $x$

$$
G\left(\pi_{n}, x\right) \leqq\left\{G\left(\pi_{n}, x\right)-g\left(\pi_{n}, \pi_{n}^{*}, x\right)\right\}+\epsilon / 2,
$$$$
n \geqq n_{\epsilon, \mathbf{x}} .
$$

But for all $n$ there exists $\pi_{n}^{*}$ such that $G\left(\pi_{n}, x\right)-g\left(\pi_{n}, \pi_{n}^{*}, x\right)<\epsilon / 2$. And since (2) holds for all valuations $\pi_{n}^{*}$ we must have $G\left(\pi_{n}, x\right)<\epsilon$ for $n \geqq n_{\epsilon, x}$. Hence

$$
\lim _{n \rightarrow \infty} G\left(\pi_{n}, x\right)=0 .
$$

The sequence of functions $G\left(\pi_{n}, x\right)$ satisfies the hypothesis of Lebesgue's theorem on bounded convergence. Applying this theorem we get

$$
\lim _{n \rightarrow \infty} \int_{a}^{b} G\left(\pi_{n}, x\right) d x=0 .
$$

But since $0 \leqq g\left(\pi_{n}, \pi_{n}^{*}, x\right) \leqq G\left(\pi_{n}, x\right)$, it follows that

$$
\lim _{n \rightarrow \infty} \int_{a}^{b} g\left(\pi_{n}, \pi_{n}^{*}, x\right) d x=0 .
$$

In view of (ii), however,

$$
\int_{a}^{b} g\left(\pi_{n}, \pi_{n}^{*}, x\right) d x=\sum_{k=1}^{p(n)} a_{\pi_{n}, \pi_{n}^{*}}^{k}\left|\Delta_{k}\right|,
$$

hence the theorem. (Q.E.D.)

(8.1) and (8.2) at once justify this result when limits as $\pi \downarrow$ are taken instead of sequential limits. We can therefore state the result of the last lemma in the following more convenient form.

Lemma A1.2. If (i) for all $\pi$ and all valuations $\pi^{*}, g\left(\pi, \pi^{*}, x\right)$ is a positive step-function on $[a, b]$ such that for all $x \in \Delta_{k} \in \pi, g\left(\pi, \pi^{*}, x\right)=a_{\pi, \pi}^{k}$, a positive constant;

(ii) $g\left(\pi, \pi^{*}, x\right)$ is uniformly bounded for all $x \in[a, b]$ and all $\pi, \pi^{*}$, that is, $g\left(\pi, \pi^{*}, x\right)<K$ then

(iii) $\lim _{\pi \downarrow} g\left(\pi, \pi^{*}, x\right)=0$, for all $x \in[a, b]$;

$$
\lim _{\pi \downarrow} \sum_{k=1}^{n(\pi)} a_{\pi, \pi^{*}}^{k}\left|\Delta_{k}\right|=0 .
$$

\section{ApPendix II. An EXTENSION OF TANNERY's THEOREM}

The derivation of the Peano series for the product integral is considerably simplified by the following lemma, which is an extension to directed sets of a result due to Tannery (cf. T. Bromwich [1, pp. 136-137]).

Lemma A2. If (i) for all $\pi, \pi^{*}$ and all positive integers $r \leqq n(\pi)$, $J_{r}\left(\pi, \pi^{*}\right) \in X$ 
(ii) for each $r, \lim _{\pi \downarrow} J_{r}\left(\pi, \pi^{*}\right)$ exists;

(iii) for each $r, J_{r}\left(\pi, \pi^{*}\right)$ is bounded, and

$$
M_{r}=\sup _{\pi, \pi^{*} ; n(\pi) \geqq r}\left|J_{r}\left(\pi, \pi^{*}\right)\right| ;
$$

(iv) $\sum_{1}^{\infty} M_{r}$ converges;

then

$$
\begin{aligned}
\lim _{\pi \downarrow}\left[J_{1}\left(\pi, \pi^{*}\right)\right. & \left.+J_{2}\left(\pi, \pi^{*}\right)+\cdots+J_{n(\pi)}\left(\pi, \pi^{*}\right)\right] \\
& =\lim _{x \downarrow} J_{1}\left(\pi, \pi^{*}\right)+\lim _{\pi \downarrow} J_{2}\left(\pi, \pi^{*}\right)+\cdots+\lim _{\pi \downarrow} J_{r}\left(\pi, \pi^{*}\right)+\cdots,
\end{aligned}
$$

the series on the R.H.S. being convergent.

Proof. Let

$$
\begin{aligned}
F\left(\pi, \pi^{*}\right) & =J_{1}\left(\pi, \pi^{*}\right)+\cdots+J_{n(\pi)}\left(\pi, \pi^{*}\right), \\
w_{r} & =\lim _{\boldsymbol{\tau}} J_{r}\left(\pi, \pi^{*}\right) .
\end{aligned}
$$

Then $\left|w_{r}\right| \leqq M_{r}$. Therefore $\sum_{1}^{\infty} w_{r}=w$ converges absolutely. We must show that $F\left(\pi, \pi^{*}\right)$ approaches $w$ as $\pi \downarrow$. Let $\epsilon>0$ be given. There exists a positive integer $m_{\epsilon}$ such that for all $n>m \geqq m_{\epsilon}, \sum_{m}^{n} M_{r}<\epsilon / 3$. Hence

$$
\left|\sum_{r=m_{e}+1}^{\infty} w_{r}\right| \leqq \sum_{r=m_{e}+1}^{\infty} M_{r}<\epsilon / 3,
$$

and for all $\pi$ such that $n(\pi)>m$, we have

$$
\left|\sum_{r=m_{\epsilon}+1}^{n(\pi)} J_{r}\left(\pi, \pi^{*}\right)\right| \leqq \sum_{r=m_{e}+1}^{n(\pi)} M_{r}<\epsilon / 3
$$

Hence for all $\pi$ with $n(\pi)>m$ we have

$$
\begin{aligned}
\left|F\left(\pi, \pi^{*}\right)-w\right| & =\left|\sum_{r=1}^{m_{e}} J_{r}\left(\pi, \pi^{*}\right)+\sum_{r=m_{e}+1}^{n(\pi)} J_{r}\left(\pi, \pi^{*}\right)-\sum_{r=1}^{m_{e}} w_{r}-\sum_{r=m_{e}}^{\infty} w_{r}\right| \\
& \leqq \sum_{r=1}^{m_{e}}\left|J_{r}\left(\pi, \pi^{*}\right)-w_{r}\right|+\left|\sum_{r=m_{e}+1}^{n(\pi)} J_{r}\left(\pi, \pi^{*}\right)\right|+\left|\sum_{r=m_{e}+1}^{\infty} w_{r}\right| \\
& \leqq \sum_{r=1}^{m_{e}}\left|J_{r}\left(\pi, \pi^{*}\right)-w_{r}\right|+2 \epsilon / 3,
\end{aligned}
$$

from (1) and (2). But since $\lim _{\pi \downarrow} J_{r}\left(\pi, \pi^{*}\right)=w_{r}$, there exists $\pi_{\epsilon}$ with $n\left(\pi_{\epsilon}\right)>m_{\text {. }}$ such that for all $\pi<\pi_{\epsilon}$ and all valuations $\pi^{*}$

$$
\sum_{r=1}^{m_{\epsilon}}\left|J_{r}\left(\pi, \pi^{*}\right)-w_{r}\right|<\epsilon / 3 \text {. }
$$


It follows from (3) that $\pi \prec \pi_{\epsilon}$ implies $\left|F\left(\pi, \pi^{*}\right)-w\right|<\epsilon$. Hence

$$
\lim _{\pi \downarrow} F\left(\pi, \pi^{*}\right)=w
$$

and the proof is completed. (Q.E.D.)

\section{BIBLIOGRAPHY}

\section{S. BANACH}

1. Theorie des operations linéaires, Warsaw, 1932.

GARRETT BirKhoFF

1. Integration of functions with values in a Banach space, Trans. Amer. Math. Soc. vol. 38 (1935) pp. 357-378.

2. On product integration, Journal of Mathematics and Physics (Massachusetts Institute of Technology) vol. 16 (1937) pp. 104-132.

3. Lattice theory, Amer. Math. Soc. Colloquium Publications, vol. 25, New York, 1940.

G. D. Birkhoff AND R. E. LANGER

1. The boundary problems and developments associated with a system of ordinary linear differential equations of the first order, Proceedings of the American Academy of Arts and Sciences vol. 58 (1923) pp. 49-128.

T. BROMWICH

1. An introduction to the theory of infinite series, London, 1931.

M. FRECHET

1. Sur quelques points du calcul fonctionnel, Rend. Circ. Mat. Palermo vol. 22 (1906) pp. 1-74.

1. Gelfand

1. Normierte Ringe, Rec. Math. (Mat. Sbornik) N.S. vol. 9 (1941) pp. 3-24.

E. Goursat

1. A course of mathematical analysis, vol. 1 (translation by E. R. Hedrick), Boston, 1917.

L. M. Graves

1. Riemann integration and Taylor's theorem in general analysis, Trans. Amer. Math. Soc. vol. 29 (1927) pp. 163-177.

E. W. HoBson

1. The theory of functions of a real variable, vol. 1, Cambridge, 1927.

E. H. Moore AND H. L. SMith

G. Peano

1. A general theory of limits, Amer. J. Math. vol. 44 (1922) pp. 102-121.

1. Intégration par sêries des équations differentielles linéaires, Math. Ann. vol. 32 (1888)

G. RASCH pp. $450-456$.

1. Zur Theorie und Anwendung des Productintegrals, J. Reine Angew. Math. vol. 171 (1934) pp. 65-119.

\section{SCHLESINGER}

1. Neue Grundlage für einen Infinitesimalkalkul der Matrizen, Math. Zeit. vol. 33 (1931) pp. 33-61.

2. Weitere Beiträge zum Infinitesimalkalkul der Matrizen, Math. Zeit. vol. 35 (1932) pp. 485501.

V. VOLTERRA

1. Sulle equazioni differzeniali lineari, Rendiconto Accademia dei Lincei vol. 3 (1887) pp. 393-396.

2, 3. Sui fondamenti della teoria della equazioni differenziali, Memorie Sociètà Italiana delle scienze (3) vol. 6 (1887) p. 6; (3) vol. 12 (1902) pp. 3-68. 


\section{VolterRa AND B. HostinsKy}

1. Operations infinilisimales lineaires, Paris, 1938.

J. VON NEUMANN

1. Über die analytischen Eigenschaften von Gruppen linearer Transformationen und ihre Darstellungen, Math. Zeit. vol. 30 (1929) pp. 3-42.

J. H. M. WEDDERBURN

1. The absolute value of the product of two matrices, Bull. Amer. Math. Soc. vol. 31 (1925) pp. 304-308.

K. YosidA

1. On the groutp embedded in a metrical complete ring, Jap. J. Math. vol. 13 (1936) pp. 7-26.

HARVARD UNIVERSITY,

Cambridge, Mass. 